\title{
Infinitely many coexisting strange attractors
}

\author{
by \\ Eduardo COLLI \\ Instituto de Matemática Pura e Aplicada (IMPA), \\ Est. D. Castorina, 110, 22460-320, Rio de Janeiro, RJ, Brasil. \\ E-mail: ecolli@impa.br
}

\begin{abstract}
We prove that $C^{\infty}$ diffeomorphisms of a two-dimension manifold $M$ with a homoclinic tangency are in the closure of an open set of Diff ${ }^{\infty}(M)$ containing a dense subset of diffeomorphisms exhibiting infinitely many coexisting Hénon-like strange attractors (or repellers). A similar statement is posed in terms of one-parameter $C^{\infty}$ families of diffeomorphisms unfolding a homoclinic tangency. Moreover, we show the existence of infinitely many dynamical phenomena others than strange attractors.

(C) 1998 L'Association Publications de l'Institut Henri Poincaré. Published by Elsevier B.V. All rights reserved

RÉSUMÉ. - Nous considérons les difféomorphismes $C^{\infty}$ d'une variété bidimensionnelle $M$ qui exhibent une tangence homoclinique. Nous démontrons qu'ils appartiennent à la fermeture d'un ensemble ouvert de Diff $^{\infty}(M)$ admettant un sous-ensemble dense de difféomorphisme exhibant une infinité d'attracteurs ou de répulseurs étranges de type Hénon. Nous énonçons un résultat similaire en termes de familles $C^{\infty}$ à un paramètre de difféomorphismes présentant une tangence homoclinique. De même nous montrons l'existence d'une infinité d'autres phénomènes dynamiques à côté des attracteurs étranges.

(C) 1998 L'Association Publications de l'Institut Henri Poincaré. Published by Elsevier B.V. All rights reserved
\end{abstract}

\section{INTRODUCTION}

Homoclinic behavior, corresponding to possible intersections of the stable and unstable manifolds of some orbit, was first introduced by Poincaré about a century ago [10]. He suggested that deep dynamic phenomena should be

Annales de l'Institut Henri Poincaré - Analyse non linéaire - 0294-1449 Vol. 15/98/05/

(C) 1998 L'Association Publications de l'Institut Henri Poincaré. Published by Elsevier B.V. All rights reserved 
involved in the presence of such a behavior. In the present work we exhibit one more of these rich dynamic phenomena, namely the possible coexistence of infinitely many strange attractors when unfolding homoclinic tangencies.

In 1970 [5], Newhouse proved that there is an open set $\mathcal{U} \subset \operatorname{Diff}^{r}(M)$, $r>1, M$ closed and $\operatorname{dim}(M)=2$, in which the set of diffeomorphisms exhibiting a homoclinic tangency is dense. This result was a negative answer to the question that Axiom A (or hyperbolic) diffeomorphisms could be dense in the space of surface diffeomorphisms (in fact, it is still an open question in $\operatorname{Diff}^{1}(M)$ with the $C^{1}$ topology). It also implicd, in the dissipative case, the existence of a residual (Baire's second cathegory) subset $\mathcal{R}$ of $\mathcal{U}$ such that each diffeomorphism in $\mathcal{R}$ exhibits infinitely many sinks [6], as an easy consequence of the known fact that homoclinic tangencies can be approximated by sinks in the space of diffeomorphisms. In 1979 [7], Newhouse showed that such open sets actually appear arbitrarily near any diffeomorphism which has a homoclinic tangency; new and perhaps clearer proofs of Newhouse's results are presented in the book of Palis and Takens [12]. The wish to grasp some meaningful description of the "majority" of dynamical systems led Palis to conjecture that the diffeomorphisms exhibiting a homoclinic tangency could be dense in the interior of the whole complement of the hyperbolic ones, not only in the open sets described by Newhouse. In fact, these and other results mentioned below justify Palis' view that the unfolding of homoclinic tangencies might be a main bifurcating mechanism [12].

In the 80's and 90's there was intense research done on the unfolding of homoclinic tangencies. Particularly, it has been shown that in addition to infinitely many sinks, homoclinic tangencies are approximated by critical saddle-node bifurcations, as observed by L. Mora, cascades of period doubling [18] and specially Hénon-like strange attractors [1], [9], [17], among others. All these phenomena are related to different aspects of nonhyperbolicity or even to different ways to depart from hyperbolicity. These results altogether suggest a kind of "homogeneity" in the interior of the hyperbolic diffeomorphisms complement, i.e. any nonhyperbolic phenomenon above mentioned could be approximated by all the remaining ones, as in the case of homoclinic tangencies. Indeed, this conjecture has been partially proved in the recent years by several authors. We already know that critical saddle-node bifurcations are approximated by homoclinic tangencies [8], the same for some relevant cases of period doubling bifurcations [2] and for Hénon-like strange attractors [16]. However, whether the phenomenon of infinitely many sinks can exist isolated from the other main bifurcating mechanisms is, as yet, nearly completely unknown. 
In addition to the conjectures above, Palis also proposed the existence of infinitely many coexisting strange attractors near homoclinic tangencies. The problem, although simply stated, revealed itself quite complicate since Hénon-like strange attractors do not have a key property of hyperbolic periodic point attractors, namely the stability under perturbations. In this direction, some particular results have been found. In 1990 [3], GambaudoTresser constructed an example of a $C^{2}$ diffeomorphism in the 2-disk exhibiting infinitely many hyperbolic strange attractors. However, the method of construction, which consists of gluing copies of a single attractor, does not obtain $C^{r}$ diffeomorphisms for $r>2$. Later on, in 1995, the author and F. Jorge Moreira observed that the method yields the construction of infinitely many Hénon-like strange attractors, and even infinite copies of many other dynamical phenomena, but always with stringent restriction on the differentiability of the resulting diffeomorphism. Finally, in 1994 [11], Pumariño-Rodriguez exhibited a very specific $C^{\infty}$ family of vector fields in $\mathbf{R}^{3}$, related to a saddle-focus connection, which has at least one parameter value with infinitely many Hénon-like strange attractors.

In the present work, we give an answer to the question in the $C^{\infty}$ topology and in much generality in the context of unfoldings of homoclinic tangencies of surface diffeomorphisms.

Let $M$ be a compact manifold of dimension two.

THEOREM A. - Let $f_{0} \in$ Diff $^{\infty}(M)$ be such that $f_{0}$ has a homoclinic tangency between the stable and unstable manifolds of a dissipative hyperbolic saddle $p_{0}$. Then, there exists an open set $\mathcal{V} \subset$ Diff ${ }^{\infty}(M)$ sach that

- $f_{0} \in \overline{\mathcal{V}}$;

- there exists a dense subset $\mathcal{D} \subset \mathcal{V}$ such that for all $f \in \mathcal{D}, f$ exhibits infinitely many coexisting Hénon-like strange attractors.

The open set $\mathcal{V}$ of Theorem A will be constructed as an union of open sets $\mathcal{W}_{n}$, each $\mathcal{W}_{n}$ written as

$$
\mathcal{W}_{n}=\left\{g_{\mu} ; G=\left(g_{\mu}\right)_{\mu} \in \mathcal{Z}_{n}, \mu \in I_{n}\right\},
$$

where $\mathcal{Z}_{n}$ is an open set in the space of $C^{\infty}$ one-parameter families and $I_{n}$ is an interval. In Section 6, we prove the following statement: "there is a residual subset $\mathcal{R}_{n} \subset \mathcal{Z}_{n}$ such that for each family $G=\left(g_{\mu}\right)_{\mu} \in \mathcal{R}_{n}$ there is a dense set $D_{n} \subset I_{n}$ such that for each $\mu \in D_{n}, g_{\mu}$ exhibits infinitely many coexisting Hénon-like strange attractors." Therefore

$$
\mathcal{D}=\bigcup_{n}\left\{g_{\mu} ; G=\left(g_{\mu}\right)_{\mu} \in \mathcal{R}_{n}, \mu \in D_{n}\right\}
$$

Vol. $15, n^{\circ} 5-1998$ 
fits the conclusion of Theorem A. The same statement will easily imply the following theorem.

THEOREM B. - Among the families that unfold a homoclinic tangency at parameter value 0 there is a residual subset such that if $F=\left(f_{\mu}\right)_{\mu}$ is a family in this subset, then there are intervals $I_{n} \rightarrow 0$ and dense subsets $D_{n} \subset I_{n}$ such that for $\mu \in D_{n}, f_{\mu}$ exhibits infinitely many coexisting Hénon-like strange attractors.

Besides sinks and strange attractors, we also consider codimensionone phenomena of the quadratic family $\Psi=\left(\psi_{a}\right)_{a}$, where $\psi_{a}(x, y)=$ $\left(1-a x^{2}, 0\right)$. Examples of codimension-one phenomena of the quadratic family are saddle-nodes, critical saddle-nodes, flip bifurcations, homoclinic tangencies and, although not proven in full generality, Feigenbaum attractors. Eventhough Theorem $\mathrm{A}$ is stated for Hénon-like strange attractors, yet the following theorem is a Corollary of the proof of Theorem A.

THEOREM C. - Let $\Sigma$ be a codimension-one phenomenon of the quadratic family. Under the same hypothesis of Theorem A, the resulting open set $\mathcal{V} \subset \operatorname{Diff}^{\infty}(M)$ of the conclusion also satisfies: There is a dense subset of $\mathcal{V}$ such that for each $f$ in this subset, $f$ exhibits infinitely many coexisting phenomena of $\Sigma$ type.

An important open question on the subject concerns the measure prevalence of diffeomorphisms with infinitely many attractors (periodic or not) in families with a finite number of parameters. In other words, let $F=\left(f_{\mu}\right)_{\mu \in R^{k}}$ be a $k$-parameter family of diffeomorphisms and let $P_{F} \subset \mathbf{R}^{k}$ be the set of parameters such that for $\mu \in P_{F}, f_{\mu}$ has infinitely many attractors. Is the Lebesgue measure of $P_{F}$ positive for any or for "most" families $F$ ? It is already known [13] (see also [12], Appendix 4) that for generic one-parameter families $F, P_{F}$ contains a residual subset, in the case of periodic attractors, but nothing is known about its measure. For strange attractors, Theorem B provides a similar result for a residual set of such families.

This work is organized as follows. In Section 2 we give a full account of the main results on Cantor sets used to prove the theorems and state the "Linking Lemma", which is crucial to the argument. In Section 3 we review the construction used to prove Newhouse's theorem on infinitely many sinks as presented in [12], taking especial care with the expansion and contraction rates of the basic sets involved. Section 3 can be summarized by Proposition 3.7 and the remark following it. In Section 4 we perform a renormalization scheme in 2 -cycles of periodic points with a heteroclinic 
tangency. This renormalization is needed to the control of orbits in Section 6 , which is in turn essential to guarantee space for arbitrarily small $C^{r}$ perturbations, for any $r \geq 0$. The calculations to prove convergence of the renormalization scheme in Lemma 4.1 are somewhat straightforward, but depend nontrivially on delicate relations between the eigenvalues of the periodic points involved and the amount of time spent near the periodic points. A necessary assumption to perform renormalization is the existence of linearizing coordinates in a neighbourhood of the periodic points, so at the end of Section 4 we make a delicate discussion on how to perturb the families to obtain linearizability, in a way that will be useful to the arguments of Section 6. Simpler aproaches of this question were tried without success, cven renormalization with no linearizing hypotheses. In Section 5 we make a brief summary of the theorems in [9], [17] and derive some consequences of its proof. Finally, in Section 6, we present the proof of Theorems A, B and C, after achieving the desired control on the orbits of the strange attractors.

\section{CANTOR SETS}

In this section we recall some concepts about Cantor sets in the line and their relation with dynamics. Most concepts can be found in [12]. At the end of the section we state and prove what we call the Linking Lemma.

A Cantor set here is a compact, perfect and totally disconnected set in the line. Let $K$ be a Cantor set and $I$ its convex hull. A presentation of $K$ is an ordering $\mathcal{U}=\left\{U^{(n)}\right\}_{n \geq 1}$ of the bounded gaps of $K$. An ordered presentation of $K$ is a presentation $\mathcal{U}$ such that $\left|U^{(n)}\right| \leq\left|U^{(m)}\right|$ for all $n>m$. The bridge at $u \in \partial U^{(n)}, U^{(n)} \in \mathcal{U}$, is the component $C$ of $I-\left(U^{(1)} \cup U^{(2)} \cup \ldots \cup U^{(n)}\right)$ that contains $u$. The thickness of $K$ is the number

$$
\tau(K)=\inf _{u} \tau(K, \mathcal{U}, u),
$$

where $\mathcal{U}$ is any ordered presentation of $K$,

$$
\tau(K, \mathcal{U}, u)=\frac{|C|}{\left|U^{(n)}\right|},
$$

and where $C$ is the bridge at $u \in \partial U^{(n)}$. This definition of thickness makes sense since $\tau(K)$ does not depend on the ordered presentation $\mathcal{U}$ (see [12]). Also, it is immediate to see from the definition that if $C$ is a bridge, then $\tau(C \cap K) \geq \tau(K)$.

Vol. 15, $\pi^{\circ}$ 5-1998. 
Let $K_{1}, K_{2}$ be Cantor sets and $I_{1}, I_{2}$ their convex hulls. We say that the pair $\left\langle K_{1}, K_{2}\right\rangle$ is linked if $I_{1} \cap I_{2} \neq \emptyset, I_{1}$ is not inside a gap of $K_{2}$ and $I_{2}$ is not inside a gap of $K_{1}$. If the same conditions are verified by the interiors $\stackrel{\circ}{I}_{1}, \stackrel{\circ}{I}_{2}$ of $I_{1}, I_{2}$ then the link is said to he stable.

We say that $\left\langle K_{1}, K_{2}\right\rangle$ has a sublink if there are proper intervals $C_{1} \subset I_{1}$ and $C_{2} \subset I_{2}$, bridges of $K_{1}$ and $K_{2}$, respectively, such that the pair $\left\langle C_{1} \cap K_{1}, C_{2} \cap K_{2}\right\rangle$ is linked. Finally, we say that $\left\langle K_{1}, K_{2}\right\rangle$ has two sublinks if there are two pairs of distinct proper subintervals forming independent sublinks (we will eventually say $\left\langle C_{1}, C_{2}\right\rangle$ instead of $\left\langle C_{1} \cap K_{1}, C_{2} \cap K_{2}\right\rangle$ where the full notation could be somewhat heavy).

Proposition 2.1 (Newhouse's Gap Lemma). - If $\tau\left(K_{1}\right) \cdot \tau\left(K_{2}\right)>1$ and $\left\langle K_{1}, K_{2}\right\rangle$ is linked, then $K_{1} \cap K_{2} \neq \emptyset$.

Let $I_{1}, \ldots I_{l}$ be a collection of disjoint closed intervals. Let $\Psi$ be a $C^{1+\epsilon}$ function defined in a neighborhood of each $I_{i}, i=1, \ldots, l$, such that for each $1 \leq i \leq l, \Psi\left(I_{i}\right)$ is an interval which is the convex hull of a subcollection of intervals $\left\{I_{j}, I_{j+1}, \ldots, I_{k}\right\}, 1 \leq j, k \leq l$. Suppose that $\Psi$ is expanding, i.e. $\inf _{x}\left|\Psi^{\prime}(x)\right|>1$. The set

$$
K=\bigcap_{n=0}^{\infty} \Psi^{-n}\left(I_{1} \cup \ldots \cup I_{l}\right)
$$

is a dynamically defined Cantor set, and the collection of intervals $\left\{I_{1}, \ldots, I_{l}\right\}$ is the Markov partition of $K$. A further property is often required: For $n$ sufficiently large, $\Psi\left(K \cap I_{i}\right)=K, 1 \leq i \leq l$, meaning that $\left.\Psi\right|_{K}$ is topologically mixing.

If $f$ is a $C^{2}$ diffeomorphism on a manifold of dimension 2 and $\Lambda$ is a hyperbolic set of saddle type (a horseshoe), then $W_{\text {loc }}^{s}(x) \cap \Lambda$ and $W_{\text {loc }}^{u}(x) \cap \Lambda$ are dynamically defined Cantor sets (see [12]).

Propostrion 2.2. - If $K$ is a dynamically defined Cantor set, then $0<\tau(K)<\infty$.

Let $k \in K$. Define

$$
\tau_{\text {loc }}(K, k)=\lim _{\epsilon \rightarrow 0}\left(\sup \left\{\tau(\tilde{K}) ; \tilde{K} \subset K \cap B_{\epsilon}(k) \text { a Cantor set }\right\}\right),
$$

the local thickness of $K$ at $k$.

Proposition 2.3. - If $K$ is a dynamically defined Cantor set, $\phi: \mathbf{R} \rightarrow \mathbf{R}$ is a $C^{1+\alpha}$ diffeomorphism and $c=\max \left|\phi^{\prime}\right| / \min \left|\phi^{\prime}\right|$, then

1. $\phi(K)$ is a dynamically defined Cantor set; 
2. $c^{-1} \tau(K) \leq \tau(\phi(K)) \leq c \tau(K)$;

3. $\tau_{\text {loc }}(\phi(K), \phi(k))=\tau_{\text {loc }}(K, k)$.

A consequence of the proposition above is that for dynamically defined Cantor sets, local thickness is independent of the point $k$. But in general $\tau_{\text {loc }}(K)>\tau(K)$.

It is possible to define a topology on dynamically defined Cantor sets in such a way that thickness and local thickness are continuous functions of $K$. We say that $\tilde{K}$ is near $K$ if $\tilde{K}$ has Markov partition $\left\{\tilde{I}_{1}, \ldots, \tilde{I}_{l}\right\}$ such that

1. the endpoints of $\tilde{I}_{1}, \ldots, \tilde{I}_{l}$ are near the corresponding endpoints of $I_{1}, \ldots, I_{l}$;

2. the function $\tilde{\Psi}$ of the definition of $\tilde{K}$ is $C^{1}$ near $\Psi$;

3. $\Psi$ is $C^{1+\epsilon}$ with Hölder constant $C, \tilde{\Psi}$ is $C^{1+\tilde{\epsilon}}$ with Hölder constant $\tilde{C}$ and $(\epsilon, C)$ is near $(\tilde{\epsilon}, \tilde{C})$.

It is not difficult to prove that if $\tilde{K}$ is sufficiently near $K$ then there exists a homeomorphism $h: K \rightarrow \tilde{K} C^{0}$-close to the identity such that $\tilde{\Psi} \circ h=h \circ \Psi$.

THEOREM 2.4. - Thickness of $K$ depends continuously on $K$.

From the proof, it can be seen that

- local thickness is also continuous in this sense;

- the continuity is uniform over all sub-Cantor sets of $K$; in particular, given $t>0$, if $\tilde{K}$ is sufficiently near $K$ then for any bridge $C$ of $K$ we have

$$
\tau(h(C) \cap \tilde{K}) \in[\tau(C \cap K)-t, \tau(C \cap K)+t] .
$$

Now we state and prove a lemma which will be used later in Section 6 . Since the hypotheses are in complicated form, in order to fulfill the requirements of the main theorem, we also state a corollary which is the simplified and intuitive version of the lemma.

Lemma 2.5 (Linking Lemma). - Let $K_{1}$ and $K_{2}$ be Cantor sets with $\tau\left(K_{1}\right) \cdot \tau\left(K_{2}\right) \geq 1+t$, for some $t>0$, and $I_{1}, I_{2}$ the convex hulls of $K_{1}$, $K_{2}$. Let $\vartheta_{\beta}^{(1)}: I_{1} \rightarrow \mathbf{R}$ and $\vartheta_{\beta}^{(2)}: I_{2} \rightarrow \mathbf{R}, \beta \in \mathbf{R}$, be such that

1. $\vartheta_{\beta}^{(i)}$ is a topological embedding, $\forall \beta \in \mathbf{R}, i=1,2$;

2. $\vartheta_{\beta}^{(i)}(x)$ is differentiable with respect to $\beta, \forall x \in K_{i}, i=1,2$;

3. $\partial_{\beta}\left(\vartheta_{\beta}^{(1)}(x)-\vartheta_{\beta}^{(2)}(y)\right) \geq c>0, \forall x \in K_{1}, y \in K_{2}$; 
4. if $\tilde{K}_{1} \subset K_{1}$ and $\tilde{K}_{2} \subset K_{2}$ are Cantor subsets with $\tau\left(\tilde{K}_{1}\right) \cdot \tau\left(\tilde{K}_{2}\right) \geq$ $1+t$, then

$$
\tau\left(\vartheta_{\beta}^{(1)}\left(\tilde{K}_{1}\right)\right) \cdot \tau\left(\vartheta_{\beta}^{(2)}\left(\tilde{K}_{2}\right)\right) \geq 1+\frac{t}{2}>1
$$

Let $\beta_{0} \in \mathbf{R}$ be such that the pair $\left\langle\vartheta_{\beta_{0}}^{(1)}\left(K_{1}\right), \vartheta_{\beta_{0}}^{(2)}\left(K_{2}\right)\right\rangle$ is linked. Then, for any $\epsilon>0$, there is $\beta$ such that

- $\left|\beta-\beta_{0}\right|<\epsilon$;

- $\left\langle\vartheta_{\beta}^{(1)}\left(K_{1}\right), \vartheta_{\beta}^{(2)}\left(K_{2}\right)\right\rangle$ has two (stable) sublinks.

Proof. - Consider the bounded intervals $J \subset \mathbf{R}$ such that for $\beta \in J$ the pair of Cantor sets $\left\langle\vartheta_{\beta}^{(1)}\left(K_{1}\right), \vartheta_{\beta}^{(2)}\left(K_{2}\right)\right\rangle$ is linked, and fix the interval $J_{0}$ to which $\beta_{0}$ belongs. By the Gap Lemma (Proposition 2.1), there are bridges $C_{1}$ of $K_{1}$ and $C_{2}$ of $K_{2}$ such that

a. $\left|\vartheta_{\beta}^{(1)}\left(C_{1}\right)\right|,\left|\vartheta_{\beta}^{(2)}\left(C_{2}\right)\right|<\epsilon / 3 c$ and $\left|\vartheta_{\beta}^{(2)}\left(C_{2}\right)\right| \leq\left|\vartheta_{\beta}^{(1)}\left(C_{1}\right)\right|, \forall \beta \in J_{0}$;

b. $\left\langle\vartheta_{\beta_{0}}^{(1)}\left(C_{1} \cap K_{1}\right), \vartheta_{\beta_{0}}^{(2)}\left(C_{2} \cap K_{2}\right)\right\rangle$ is a linked pair.

Let $U_{2}$ be one of the greatest gaps of $C_{2}$ and $Q_{2}, \tilde{Q}_{2}$ the adjacent left and right bridges. By hypothesis 3, there is $\beta_{1}$ with $\left|\beta_{1}-\beta_{0}\right|<2 \epsilon / 3$ such that the right endpoint of $\vartheta_{\beta_{1}}^{(2)}\left(Q_{2}\right)$ coincides with the left endpoint of $\vartheta_{\beta_{1}}^{(1)}\left(C_{1}\right)$ (see Figure 1). Suppose that $\vartheta_{\beta_{1}}^{(2)}\left(\tilde{Q}_{2}\right)$ is contained in $\overline{\vartheta_{\beta_{1}}^{(1)}\left(U_{1}\right)}$, where $U_{1}$ is a bounded gap of $K_{1}$ ( $U_{1}$ could not be an unbounded gap by a.). Let $Q_{1}$ be the left component of $C_{1} \backslash U_{1}$. Hence $\vartheta_{\beta_{1}}^{(1)}\left(Q_{1}\right) \subset \overline{\vartheta_{\beta_{1}}^{(2)}\left(U_{2}\right)}$. Thus

$$
1 \geq \frac{\left|\vartheta_{\beta_{1}}^{(1)}\left(Q_{1}\right)\right|}{\left|\vartheta_{\beta_{1}}^{(2)}\left(U_{2}\right)\right|} \cdot \frac{\left|\vartheta_{\beta_{1}}^{(2)}\left(\tilde{Q}_{2}\right)\right|}{\left|\vartheta_{\beta_{1}}^{(1)}\left(U_{1}\right)\right|} \geq \tau\left(\vartheta_{\beta_{1}}^{(1)}\left(C_{1}\right)\right) \cdot \tau\left(\vartheta_{\beta_{1}}^{(2)}\left(C_{2}\right)\right)>1
$$

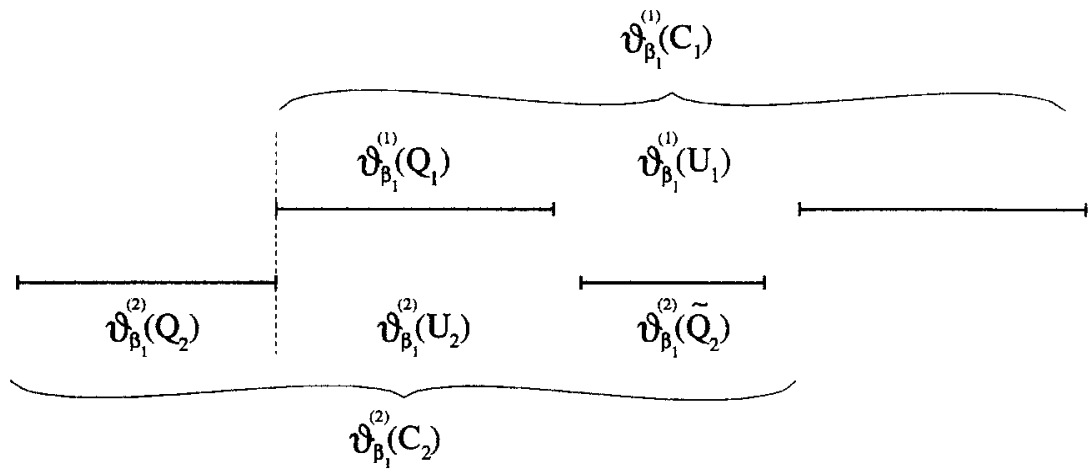

Fig. 1. - A contradiction. 
which is a contradiction. Therefore $\vartheta_{\beta_{1}}^{(2)}\left(\tilde{Q}_{2}\right)$ has a (stable) link with a bridge of $\vartheta_{\beta_{1}}^{(1)}\left(C_{1} \cap K_{1}\right)$. Without destroying this link, choose $\beta$ near $\beta_{1}$ (with $\left|\beta-\beta_{0}\right|<\epsilon$ ) such that $\vartheta_{\beta}^{(2)}\left(Q_{2}\right)$ and $\vartheta_{\beta}^{(1)}\left(Q_{1}\right)$ have a stable link.

Corollary 2.6. - Let $K_{1}$ and $K_{2}$ be Cantor sets such that $\tau\left(K_{1}\right)$. $\tau\left(K_{2}\right)>1$ and the pair $\left\langle K_{1}, K_{2}\right\rangle$ is linked. Then, given $\epsilon>0$, there is $|\beta|<\epsilon$ such that the pair $\left\langle K_{1}, K_{2}+\beta\right\rangle$ has two stable sublinks.

Corollary 2.6 had already been proved by Kraft [4] using similar arguments.

\section{THE UNFOLDING OF HOMOCLINIC TANGENCIES}

The goal of this section is to obtain Proposition 3.7, by recalling the main tools used to prove Newhouse's theorem on infinitely many sinks. We follow the ideas presented in [12] and obtain further estimates, necessary for Section 4, on contraction and expansion rates of the basic sets involved.

Let $p$ be a saddle fixed point for $f$ such that its stable manifold $W^{s}(p)$ and its unstable manifold $W^{u}(p)$ have a point of non-transversal intersection (a homoclinic tangency). Suppose dissipativeness at $p$, i.e. $|\operatorname{det} D f(p)|<1$. Otherwise, if $|\operatorname{det} D f(p)|>1$, just take $f^{-1}$; and if $|\operatorname{det} D f(p)|=1$, one can find arbitrarily near $f$ a diffeomorphism $\tilde{f}$ with $|\operatorname{det} D \tilde{f}(\tilde{p})|<1$, where $\tilde{p}$ is the continuation of the hyperbolic point $p$. Let $\lambda$ and $\sigma$ be the contractive and expanding eigenvalues of $D f(p)$. Assume without loss of generality that both are positive. Suppose that there are $C^{2}$ linearizing coordinates $(x, y)$ in a neighborhood $U$ of $p$, i.e. $f$ has the form $(x, y) \mapsto(\lambda \cdot x, \sigma \cdot y)$ in $U$. If this is not the case, there is $\tilde{f}$ arbitrarily near $f$ such that the linearizing coordinates are guaranteed, since their existence is an open and dense condition in $\operatorname{Diff}^{\infty}(M)$ (see [14], [15]). To be more specific, linearization around $p$ is possible ever since the eigenvalues $\lambda$ and $\sigma$ do not satisfy a finite number of certain equalities, often denominated resonances. By another perturbation, the point of contact between the stable and unstable manifolds can be made quadratic.

Now take a family $\left(f_{\mu}\right)_{\mu} \subset \operatorname{Diff}^{\infty}(M)$ such that $f_{0}=f$. The point $p$ has continuation $p_{\mu}$ with eigenvalues $\lambda_{\mu}$ and $\sigma_{\mu}$, which we will denote for shortness simply as $\lambda$ and $\sigma$. Up to rescaling of the linearizing coordinates we can suppose that $U$ contains $\{(x, y) ;|x| \leq 2,|y| \leq 2\}, q=(1,0)$ is the point of tangency and $u=(0,1)=f_{0}^{-N}(q)$, for some $N>0$. By openess of linearizability, $f_{\mu}$ is also linearizable in $U$ for $\mu$ small. 
We can write $f_{\mu}^{N}$ near $(0,1)$ as

$(x, y) \mapsto\left(1+\alpha(y-1)+\tilde{H}_{1}(\mu, x, y-1), \beta(y-1)^{2}+s \mu+\gamma x+\tilde{H}_{2}(\mu, x, y-1)\right)$,

where $\alpha, \beta, \gamma$ are non-zero constants (since the contact between $W^{s}(p)$ and $W^{u}(p)$ is non-degenerate) and $s \neq 0$ since we assume that the family $\left(f_{\mu}\right)_{\mu}$ generically unfolds the quadratic tangency. We assume $s=1$ and, for $\mu=x=y=0$,

$$
\left\{\begin{array}{l}
\tilde{H}_{1}=\partial_{y} \tilde{H}_{1}=0 \\
\tilde{H}_{2}=\partial_{x} \tilde{H}_{2}=\partial_{y} \tilde{H}_{2}=\partial_{\mu} \tilde{H}_{2}=\partial_{y y} \tilde{H}_{2}=0 .
\end{array}\right.
$$

Moreover, using a $\mu$-reparametrization and $\mu$-dependent linear changes of coordinates, we can suppose $\tilde{H}_{1}(\mu, 0,0) \equiv 0, \tilde{H}_{2}(\mu, 0,0) \equiv 0$ and $\partial_{y} \tilde{H}_{2}(\mu, 0,0) \equiv 0$, so that $\partial_{\mu} \tilde{H}_{1}(\mu, 0,0)=0, \partial_{y \mu} \tilde{H}_{2}(\mu, 0,0)=0$ and $\partial_{\mu \mu} \tilde{H}_{2}(\mu, 0,0)=0$.

Define the change of coordinates

$$
\left\{\begin{array}{l}
\xi=\sigma^{n}(x-1) \\
\eta=\sigma^{2 n}\left(y-\sigma^{-n}\right) \\
\nu=\sigma^{2 n}\left(\mu+\gamma \lambda^{n}-\sigma^{-n}\right)
\end{array}\right.
$$

After that, define again new coordinates

$$
\left\{\begin{array}{l}
\tilde{\xi}=\alpha^{-1} \beta \xi \\
\tilde{\eta}=\beta \eta \\
\tilde{\nu}=\beta \nu
\end{array}\right.
$$

and denote $\Theta_{n, \tilde{\nu}}$ the function taking $(\tilde{\xi}, \tilde{\eta})$ to $(x, y)$ and $M_{n}(\tilde{\nu})$ the function taking $\tilde{\nu}$ to $\mu$ (the inverses of the coordinate changes defined above).

Proposition 3.1. - Let $K$ be a compact set in the $(\tilde{\nu}, \tilde{\xi}, \tilde{\eta})$-space.

1. The images of $K$ under the maps

$$
(\tilde{\nu}, \tilde{\xi}, \tilde{\eta}) \mapsto\left(M_{n}(\tilde{\nu}), \Theta_{n, \tilde{\nu}}(\tilde{\xi}, \tilde{\eta})\right)
$$

converge, as $n \rightarrow \infty$, to $(0, q)$ in the $(\mu, x, y)$-space;

2. the domains of the maps

$$
\varphi^{(n)}:(\tilde{\nu}, \tilde{\xi}, \tilde{\eta}) \mapsto\left(\tilde{\nu}, \Theta_{n, \tilde{\nu}}^{-1} \circ f_{M_{n}(\tilde{\nu})}^{n+N} \circ \Theta_{n, \tilde{\nu}}\right)
$$

converge to $\mathbf{R}^{3}$; 
3. the maps $\left.\varphi^{(n)}\right|_{K}$ converge, in the $C^{2}$ topology, to the map

$$
\Psi:(\tilde{\nu}, \tilde{\xi}, \tilde{\eta}) \mapsto\left(\tilde{\nu}, \tilde{\eta}, \tilde{\eta}^{2}+\tilde{\nu}\right) .
$$

Proof. - See [12], Chapter 3.

The family $\Psi=\left(\psi_{\nu}\right)_{\nu}$ with $\psi_{\nu}(x, y)=\left(y, y^{2}+\nu\right)$ is equivalent to the family $(x, y) \mapsto\left(1-a x^{2}, 0\right)$, so we will use interchangeably the symbol $\Psi$ to denote both families, except when clarity requires specification. Properties of $\Psi=\left(\psi_{a}\right)_{a}$ are identically valid for $\Psi=\left(\psi_{\nu}\right)_{\nu}$ and vice-versa (we could obtain $\psi_{a}(x, y)=\left(0,1-a y^{2}\right)$ dircctly if the renormalization was done with another scaling for the variable $x$ ). We also consider the converging functions $\varphi^{(n)}$ of Proposition 3.1 as approaching $\Psi=\left(\psi_{a}\right)_{a}$.

The endomorphisms $\psi_{a}$ have two fixed points, namely $Q_{a} \in\{x<0\}$ and $P_{a} \in\{x>0\}$. For $a=2, Q_{a}=(-1,0)$, the right unstable separatrix of $Q_{a}$ is the interval $[-1,1]$ and the stable manifold of $Q_{a}$ is the vertical line $\{y=-1\}$. The situation can be regarded as $Q_{a}$ having a homoclinic tangency. If $\Phi=\left(\varphi_{a}\right)_{a}$ is a family of diffeomorphisms $C^{2}$ near $\Psi$, we have a true homoclinic tangency for $a=a(\Phi)$ involving the continuations of $Q_{a}$, $W^{s}\left(Q_{a}\right)$ and $W^{u}\left(Q_{a}\right)$. Moreover, $a(\Phi)$ approaches 2 as $\Phi$ approaches $\Psi$.

It is well known that $\psi_{2}: x \mapsto 1-2 x^{2}$ for $x \in[-1,1]$ is conjugated to the tent map $T:[-1,1] \rightarrow[-1,1]$ defined by $T(x)=1-2|x|$. The conjugacy is given by the map $J(x)=\sin \left(\frac{\pi x}{2}\right)$, i.e. $T=J^{-1} \circ \psi_{2} \circ J$.

PROPOSITION 3.2. $-T$ has arbitrarily thick invariant Cantor sets.

Proof. - Here we only indicate which are the invariant Cantor sets. The detailed proof can be seen in [12], Chapter 6. Fix $m \geq 3$ and let $q \in(-1,1)$ be the unique point of period $m$ for $T$ whose orbit $q=q_{0}, q_{1}=T(q), \ldots, q_{m}=T^{m}(q)=q$ satisfies

$$
q_{2}<q_{3}<\ldots<q_{m-1}<0<q_{m}=q_{0}<q_{1} .
$$

Define $q_{m}^{*} \in(-1,0)$ by $T\left(q_{m}^{*}\right)=T(q)$ and, for $i=3, \ldots, m-1$, $q_{i}^{*}-T^{-1}\left(q_{i+1}^{*}\right) \cap(-1,0)$. Consider the intervals

$$
I_{1}=\left[q_{2}, q_{1}\right], I_{2}=\left[q_{2}, q_{3}^{*}\right], \ldots, I_{m-1}=\left[q_{m-1}, q_{m}^{*}\right], I_{m}=\left[q_{m}, q_{1}\right] .
$$

We have

$$
I_{i} \subset I_{1}, i=2, \ldots, m
$$

and

$$
\begin{aligned}
T\left(I_{i}\right) & =I_{i+1}, i=2, \ldots, m-1, \\
T\left(I_{m}\right) & =I_{1} .
\end{aligned}
$$

Vol. 15, $\mathrm{n}^{\cup}$ 5-1998. 
Finally, define the Cantor set

$$
K_{m}=\bigcap_{i=0}^{\infty} T^{-i}\left(I_{2} \cup \ldots \cup I_{m}\right),
$$

that is a dynamically defined Cantor set. It is not difficult to see that $\tau\left(K_{m}\right) \rightarrow \infty$ as $m \rightarrow \infty$.

Observe that $K_{m} \subset(-1,0) \cup(0,1)$, so the map $T$ is differentiable in a neighborhood of $K_{m}$. Moreover, $\left.T\right|_{K_{m}}$ is uniformly expanding (vectors are multiplied by 2). Since $J$ is a diffeomorphism in a neighborhood of $K_{m},\left.T\right|_{K_{m}}$ is differentiably conjugated to $\left.\psi_{2}\right|_{J\left(K_{m}\right)}$, hence we also have $\tau_{\text {loc }}\left(J\left(K_{m}\right)\right) \rightarrow \infty$ as $m \rightarrow \infty$, by Proposition 2.3. Furthermore, there exists $N$, which depends on $m$, such that $\left.\psi_{2}^{N}\right|_{J\left(K_{m}\right)}$ is uniformly expanding.

PROPOSITION 3.3. - Let $m$ and $K_{m}$ be as above. Let $\varphi$ be a diffeomorphism $C^{2}$ sufficiently near $\psi_{2}$. Then $\varphi$ has a basic set $\tilde{K}_{m}$ which is the continuation of $J\left(K_{m}\right)$ and such that $\tau_{\text {loc }}^{s}\left(\tilde{K}_{m}\right)$ is near $\tau_{\text {loc }}\left(K_{m}\right)$.

Proof. - see [12], Chapter 6.

Let $p$ be a saddle fixed point with contractive and expanding eigenvalues $\lambda$ and $\sigma$ which generically unfolds a homoclinic tangency. Then the family $\left(\varphi_{\nu}^{(n)}\right)_{\nu}$ with

$$
\varphi_{\nu}^{(n)}=\Theta_{n, \nu}^{-1} \circ f_{M_{n}(\nu)}^{n+N} \circ \Theta_{n, \nu}
$$

defined above is $C^{2}$ near $\Psi$ if $n$ is big. Therefore, by Proposition 3.3, for fixed $m$ and $n$ both large, we have hyperbolic sets $K_{m}^{(n)}$ for $\varphi_{\nu_{n}}^{(n)}$ with $\nu_{n} \rightarrow 2$ as $n \rightarrow \infty$. Moreover, the parameter values $\nu_{n}$ can be chosen in such a way that $\varphi_{\nu_{n}}^{(n)}$ has a homoclinic tangency associated to the saddle fixed point near $(-1,0)$, see above. Denoting by $Q^{(n)}$ this saddle fixed point, it is proven in [12] that $Q^{(n)}$ is heteroclinically related to a periodic point $Q_{m}^{(n)} \in K_{m}^{(n)}$, i.e. $W^{u}\left(Q^{(n)}\right) \cap W^{s}\left(Q_{m}^{(n)}\right) \neq \emptyset$ and $W^{s}\left(Q^{(n)}\right) \cap W^{u}\left(Q_{m}^{(n)}\right) \neq \emptyset$, both intersections transversal. Furthermore, for $\varphi=\varphi_{\nu_{n}}^{(n)}$, there are $0<\underline{\lambda}=\underline{\lambda}(n)<\bar{\lambda}=\bar{\lambda}(n)<1,1<\underline{\sigma}<\bar{\sigma}<3$ and $C=C(n)$ such that

- $C^{-1} \underline{\lambda}^{i}\|u\| \leq\left\|D \varphi^{i}(x) \cdot u\right\| \leq C \bar{\lambda}^{i}\|u\|$

- $C^{-1} \underline{\sigma}^{i}\|v\| \leq\left\|D \varphi^{i}(x) \cdot v\right\| \leq C \bar{\sigma}^{i}\|v\|$,

for all $x \in K_{m}^{(n)}, u \in E_{x}^{s}, v \in E_{x}^{u}$ and $i \geq 0$.

Thus, if $x \in K_{m}^{(n)}$ is a periodic point for $\varphi$ of period $j$, then $D \varphi^{j}(x)$ has the stable eigenvalue between $\underline{\lambda}(n)^{j}$ and $\bar{\lambda}(n)^{j}$, and the unstable eigenvalue between $\underline{\sigma}^{j}$ and $\bar{\sigma}^{j}$. Denoting

$$
\Lambda_{m}^{(n)}=\Theta_{n, \nu_{n}}\left(K_{m}^{(n)}\right)
$$


then $\Lambda_{m}^{(n)}$ is a hyperbolic set for $f_{\mu_{n}} \equiv f_{M_{n}\left(\nu_{n}\right)}$ of period $n+N$. Let $z \in \Lambda_{m}^{(n)}$ be a periodic point for $f_{\mu_{n}}$ of period $k=(n+N) j$. Then $z=\Theta_{n, \nu_{n}}(x)$, where $x$ is a periodic point for $\varphi=\varphi_{\nu_{n}}^{(n)}$ of period $j$. We conclude that if $\lambda_{1}^{k}$ and $\sigma_{1}^{k}$ are the stable and unstable eigenvalues of $D f^{n+N}(z)$, then

$$
\sigma_{1}^{k}=\sigma_{1}^{(n+N) j} \leq \bar{\sigma}^{j} \leq 3^{j}=(\sqrt[n+N]{3})^{k}
$$

which implies $\sigma_{1} \leq^{n+N} \sqrt{3}$. Also, using $\left|\operatorname{det} D f^{N}\right| \leq K$ near $f^{-N}(q), K$ a large constant, we obtain

$$
\lambda_{1}^{k}-\frac{1}{\sigma_{1}^{k}}\left|\operatorname{det} D f^{(n+N) j}(z)\right| \leq K^{j}(\sigma \lambda)^{n j},
$$

which implies $\lambda_{1} \leq \lambda_{0}<1$, for large $n$, where $\lambda_{0}$ does not depend on $n$. We conclude that by increasing $n$, it is possible to choose $\sigma_{1}$ arbitrarily near 1 and $\lambda_{1}$ bounded away from 1 .

Proposition 3.4. - Let $p$ be a dissipative periodic saddle point for $f \in \operatorname{Diff}^{\infty}(M)$ with eigenvalues $\lambda$ and $\sigma$ and such that $W^{s}(p)$ and $W^{u}(p)$ have a point of transversal intersection. Then, for any $\epsilon>0, p$ belongs to a hyperbolic set $\Lambda=\Lambda(\epsilon)$ which satisfies:

- $C^{-1}(\lambda-\epsilon)^{n}\|u\| \leq\left\|D f^{n}(x) \cdot u\right\| \leq C(\lambda+\epsilon)^{n}\|u\|$,

- $C^{-1}(\sigma-\epsilon)^{n}\|v\| \leq\left\|D f^{n}(x) \cdot v\right\| \leq C(\sigma+\epsilon)^{n}\|v\|$,

for any $x \in \Lambda, n \geq 0, u \in E_{x}^{s}, v \in E_{x}^{u}$, where $E_{x}^{s}$ and $E_{x}^{u}$ are the stable and unstable subspaces at $x$ of the hyperbolic decomposition of $T_{\Lambda} M$ and $C$ is a constant.

Proof. - The existence of $\Lambda$ is proven in [12], Chapter 2, and it is easy to see from the proof it verifies the property above.

COROLlary 3.5. - If $x \in \Lambda$ is a periodic point of period $k$, then the eigenvalues of $D f^{k}(x)$ are between $(\lambda-\epsilon)^{k}$ and $(\lambda+\epsilon)^{k}$ and between $\left(\begin{array}{ll}\sigma & c\end{array}\right)^{k}$ and $(\sigma+\epsilon)^{k}$.

Propostrion 3.6. - Let $\left(f_{\mu}\right)_{\mu}$ be a one-parameter family of diffeomorphisms as above with a quadratic homoclinic tangency $q$ at $\mu=0$ associated to a saddle $p$, and suppose it unfolds generically. Then there is a sequence $\mu_{l} \rightarrow 0$ such that $f_{\mu_{l}}$ has homoclinic tangencies $q_{\mu_{l}} \rightarrow q$ associated to $p_{\mu_{l}} \rightarrow p$. Moreover, the values $\mu_{l}$ can be chosen in such a way that the connected components of $W^{s}\left(p_{\mu_{l}}\right) \backslash\left\{p_{\mu_{l}}\right\}$ and $W^{u}\left(p_{\mu_{l}}\right) \backslash\left\{p_{\mu_{l}}\right\}$ that have a homoclinic tangency also have transverse homoclinic intersections.

Proof. - see [12], Chapter 3. 
Take a sufficiently large $l$ and consider the homoclinic tangency for $f_{\mu_{i}}$ between $W^{u}\left(p_{\mu_{l}}\right)$ and $W^{s}\left(p_{\mu_{l}}\right)$. By Proposition 3.6 there is also a transverse homoclinic intersection between the stable and unstable manifolds of $p_{\mu_{l}}$, so that, by Proposition 3.4, $p_{\mu_{l}}$ belongs to a hyperbolic set which we now call $\Lambda_{2}$. The hyperbolic set $\Lambda_{2}$ has an analytic continuation near $f_{\mu_{i}}$, so that without destroying $\Lambda_{2}$ we can unfold the homoclinic tangency at $f_{\mu_{l}}$ and obtain, by Proposition 2.2 and 3.3, a hyperbolic set $\Lambda_{1}$ such that $\tau_{\text {loc }}^{u}\left(\Lambda_{2}\right) \cdot \tau_{\text {loc }}^{s}\left(\Lambda_{1}\right)>1$, with a periodic point $Q_{1} \in \Lambda_{1}$ heteroclinically related to a point $Q$ which has a homoclinic tangency.

It is proven in [12] that the point $Q$ is heteroclinically related to the continuation of $p_{\mu_{l}}$ which we denote from now on by $Q_{2}$. After making a small perturbation and considering the whole discussion above, we obtain the situation stated in the following proposition.

Proposition 3.7. - Let $f_{0} \in$ Diff $^{\infty}(M)$ be such that $p$ is a dissipative periodic saddle point with a homoclinic tangency between its stable and unstable manifolds. Then, there is an $f \in \operatorname{Diff}^{\infty}(M) C^{\infty}$ arbitrarily near $f_{0}$ such that

1. $f$ has hyperbolic sets $\Lambda_{1}$ and $\Lambda_{2}$ with

$$
\tau_{\mathrm{loc}}^{\mathrm{s}}\left(\Lambda_{1}\right) \cdot \tau_{\mathrm{loc}}^{u}\left(\Lambda_{2}\right)>1
$$

2. there are periodic points $Q_{1} \in \Lambda_{1}$ and $Q_{2} \in \Lambda_{2}$ such that $W^{u}\left(Q_{1}\right)$ and $W^{s}\left(Q_{2}\right)$ meet transversally at $r$ and $W^{s}\left(Q_{1}\right)$ and $W^{u}\left(Q_{2}\right)$ meet quadratically at q;

3. there exists $c>0$ such that if $p_{1} \in \Lambda_{1}$ is a periodic point for $D f^{k_{1}}\left(p_{1}\right)$ with period $k_{1}$ and eigenvalues $\lambda_{1}^{k_{1}}$ (stable) and $\sigma_{1}^{k_{1}}$ (unstable), $p_{2} \in \Lambda_{2}$ is a periodic point for $D f^{k_{2}}\left(p_{2}\right)$ with period $k_{2}$ and eigenvalues $\lambda_{2}^{k_{2}}$ (stable) and $\sigma_{2}^{k_{2}}$ (unstable), then

(a) $\lambda_{1} \cdot \sigma_{1}<1$;

(b) $\sigma_{2}^{2 c} \cdot \lambda_{1}<1$;

(c) $\sigma_{1}$ is so small that $\sigma_{1} \cdot\left(\lambda_{2} \sigma_{2}\right)^{c / 2}<1$.

Remark. - Let $F=\left(f_{\mu}\right)_{\mu}$ be a $C^{\infty}$ family of diffeomorphisms such that $f=f_{0}$ has a homoclinic tangency between the stable and the unstable manifolds of a dissipative saddle point $p$. Among the families with this property, there is an open and dense subset which satisfies the following generic conditions: $C^{2}$ linearizability of the saddle, quadratic tangency at $f_{0}$ and generic unfolding as $\mu$ varies through 0 . Moreover, it is easy to see from the considerations above the following property of a residual (even open, see [12], Appendix 4) subset of these families: "There is a sequence 
$\mu_{n} \rightarrow 0$ such that $f=f_{\mu_{n}}$ have the properties stated in Proposition 3.7 and the subfamilies $\left(g_{\nu}\right)_{\nu}$ with $g_{\nu}=f_{\mu_{n}+\nu}$ generically unfold the heteroclinic tangency of item 2 ." After the Claim at 6.4 , this assertion will immediately imply Theorem B.

\section{RENORMALIZATION IN 2-CYCLES}

In this section we describe the renormalization scheme involving a 2-cycle of periodic points, i.e. points $p_{1}$ and $p_{2}$ periodic for $f$ such that $W^{u}\left(p_{1}\right)$ intersects transversally $W^{s}\left(p_{2}\right)$ and $W^{s}\left(p_{1}\right)$ has a quadratic contact with $W^{u}\left(p_{2}\right)$. We also make further assumptions on the eigenvalues of $p_{1}$ and $p_{2}$ to obtain convergence of the renormalization process.

First suppose that $p_{1}$ and $p_{2}$ have period 1 and are $C^{4}$ linearizable (we treat the other cases at the end of this section). This means that, under $C^{4}$ changes of coordinates, there are neighborhoods $U_{1}$ of $p_{1}$ and $U_{2}$ of $p_{2}$ such that the expression of $f$ in $U_{1}$ is $(x, y) \mapsto\left(\lambda_{1} x, \sigma_{1} y\right)$ and in $U_{2}$ is $(w, z) \mapsto\left(\lambda_{2} w, \sigma_{2} z\right)$. Extend the domain of the linearized coordinates along $W^{s}\left(p_{2}\right)$ and $W^{u}\left(p_{1}\right)$ in such a way that $U_{1}$ and $U_{2}$ intersect around the transversal crossing of $W^{s}\left(p_{2}\right)$ and $W^{u}\left(p_{1}\right)$. Also, extend $U_{1}$ along $W^{s}\left(p_{1}\right)$ until it meets $q$, the point of quadratic tangency. Let $U$ be a neighborhood of $q$ inside $U_{1}$ and extend $U_{2}$ along $W^{u}\left(p_{2}\right)$ until it meets $f^{-1}(U)$. We may suppose that $q=(1,0)$ in $U_{1}$-coordinates and $f^{-1}(q)=(0,1)$ in $U_{2}$-coordinates (see Figure 2).

Let $F=\left(f_{\mu}\right)_{\mu}$ be a $C^{\infty}$ family of diffeomorphisms with $f_{0}=f$. We know that $C^{4}$ linearizability is an open condition (see [14], [15]), so we have continuations $p_{1}=p_{1}(\mu), p_{2}=p_{2}(\mu)$, eigenvalues $\sigma_{1}=\sigma_{1}(\mu)$, $\lambda_{1}=\lambda_{1}(\mu), \sigma_{2}=\sigma_{2}(\mu), \lambda_{2}=\lambda_{2}(\mu)$ and linearizations at $p_{1}$ and $p_{2}$ corresponding to orthogonal multiplication by these eigenvalues. We may assume that $f$ has the following expression in a neighborhood of $(w, z)=(0,1)$ :

$$
\begin{aligned}
(w, z) \mapsto(x, y)=(1+\alpha(z-1) & +\tilde{H}_{1}(\mu, w, z-1), \beta(z-1)^{2} \\
& \left.+\gamma(\mu) w+s \mu+\tilde{H}_{2}(\mu, w, z-1)\right)
\end{aligned}
$$

where $\alpha, \beta, \gamma(0)$ and $s$ are non-zero constants,

$$
\begin{aligned}
& \tilde{H}_{1}=\partial_{z} \tilde{H}_{1}=0, \\
& \tilde{H}_{2}=\partial_{\mu} \tilde{H}_{2}=\partial_{z} \tilde{H}_{2}=\partial_{z z} \tilde{H}_{2}=0
\end{aligned}
$$

Vol. $15, n^{\circ}$ 5-1998. 


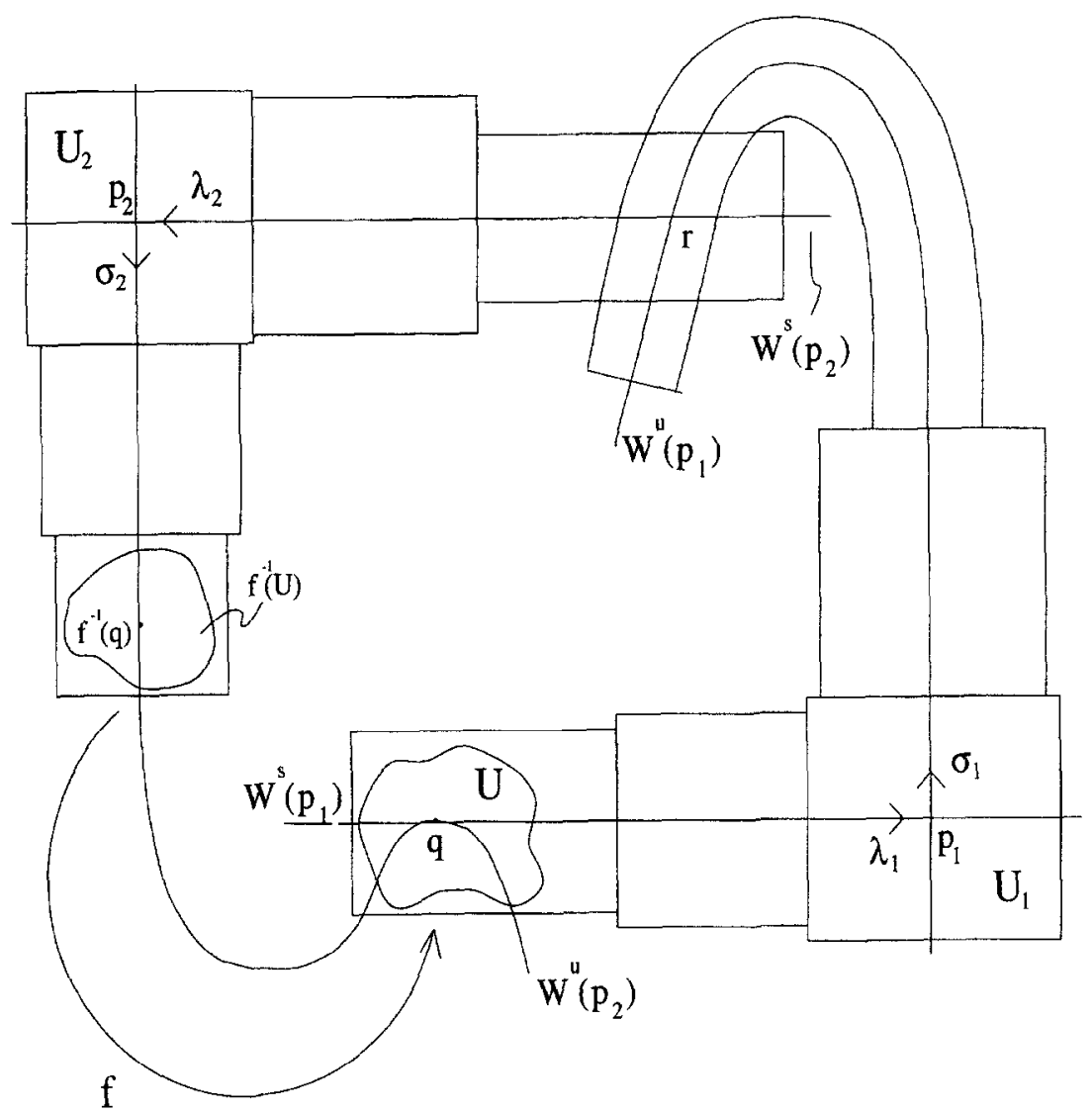

Fig. 2. - Renormalization scheme in the 2-cycle.

at $(\mu, w, z-1)=(0,0,0)$ and $\partial_{w} \tilde{H}_{2}(\mu, 0,0) \equiv 0$. Moreover, by use of a $\mu$-reparametrization and $\mu$-dependent linear changes of the space coordinates, we may even assume $s=1, \tilde{H}_{1}(\mu, 0,0) \equiv 0, \tilde{H}_{2}(\mu, 0,0) \equiv 0$ and $\partial_{z} \tilde{H}_{2}(\mu, 0,0) \equiv 0$, in such a way that $\partial_{\mu} \tilde{H}_{1}=\partial_{\mu z} \tilde{H}_{2}=\partial_{\mu \mu} \tilde{H}_{2}=0$. We still have to consider the transition map $T_{\mu}$ between $U_{1}$ and $U_{2}$ at their "transverse" intersection (see Figure 3). Suppose $T_{\mu}$ has the form $T_{\mu}(x, y)=\left(\begin{array}{l}1 \\ 0\end{array}\right)+\left(\begin{array}{cc}a_{\mu} & b_{\mu} \\ c_{\mu} & d_{\mu}\end{array}\right)\left(\begin{array}{c}x \\ y-Y_{\mu}\end{array}\right)+\left(\theta_{1}\left(\mu, x, y-Y_{\mu}\right), \theta_{2}\left(\mu, x, y-Y_{\mu}\right)\right)$,

where

$$
\begin{aligned}
& Y_{0}=1, \\
& \theta_{1}(\mu, 0,0)=\theta_{2}(\mu, 0,0)=0, \\
& \partial_{x} \theta_{1}=\partial_{y} \theta_{1}=\partial_{x} \theta_{2}=\partial_{y} \theta_{2}=0
\end{aligned}
$$


at $(\mu, 0,0)$. We may assume $Y_{\mu} \equiv 1$, also by a $\mu$-dependent linear change of coordinates. The transversality between $W^{s}\left(p_{2}\right)$ and $W^{u}\left(p_{1}\right)$ implies that $d_{\mu} \neq 0$. If one looks at $T_{\mu}^{-1}\left(W^{s}\left(p_{2}\right)\right)$ in $U_{1}$ coordinates near $(0,1)$, then it is the graph of a function $x \mapsto \Gamma_{\mu}(x)$. Analogously, $W^{u}\left(p_{1}\right)$ is the graph of a function $z \mapsto \Delta_{\mu}(z)$ in $U_{2}$ coordinates. We also define the functions $\Gamma_{\mu}^{(m)}$ and $\Delta_{\mu}^{(n)}$ whose graphs correspond to $T_{\mu}^{-1}\left(\left\{z=\sigma_{2}^{-m}\right\}\right)$ and $T_{\mu}\left(\left\{x=\lambda_{1}^{n}\right\}\right)$, respectively.

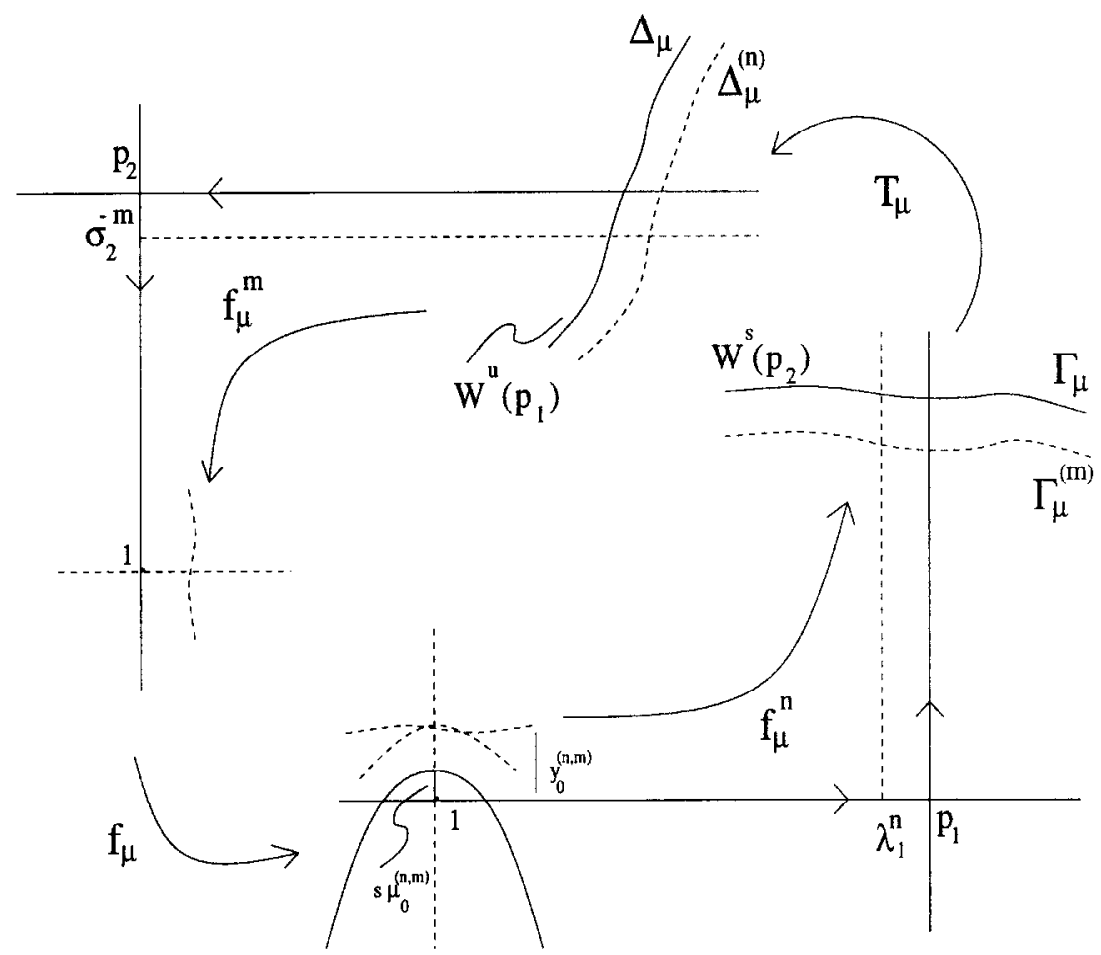

Fig. 3. - Still the renormalization scheme.

Now we are ready to define the change of coordinates. Let $\mu_{0}=\mu_{0}^{(n, m)}$ be solution of the equation

$$
\gamma\left(\mu_{0}\right) \lambda_{2}^{m} \Delta_{\mu_{0}}^{(n)}\left(\sigma_{2}^{-m}\right)+\mu_{0}=\sigma_{1}^{-n} \Gamma_{\mu_{0}}^{(m)}\left(\lambda_{1}^{n}\right) .
$$

It is easy to see that there exists such $\mu_{0}$. Let

$$
y_{0}=y_{0}^{(n, m)}(\mu)=\sigma_{1}^{-n} \Gamma_{\mu}^{(m)}\left(\lambda_{1}^{n}\right) .
$$

Vol. $15, n^{\circ} 5-1998$. 
and define

$$
\left\{\begin{array}{l}
\xi=\sigma_{1}^{n} \sigma_{2}^{m}(x-1) \\
\eta=\sigma_{1}^{2 n} \sigma_{2}^{2 m}\left(y-y_{0}\right) \\
\nu=\sigma_{1}^{2 n} \sigma_{2}^{2 m}\left(\mu-\mu_{0}\right)
\end{array}\right.
$$

In $(x, y)$-coordinates, the return map $f^{n+m+1}$ is written as

$$
\begin{aligned}
(x, y) \mapsto(1+\alpha(\bar{z}-1) & +\tilde{H}_{1}(\mu, \bar{w}, \bar{z}-1), \beta(\bar{z}-1)^{2} \\
& \left.+\gamma(\mu) \bar{w}+\mu+\tilde{H}_{2}(\mu, \bar{w}, \bar{z}-1)\right),
\end{aligned}
$$

where

$$
\bar{w}=\lambda_{2}^{m}\left(1+a_{\mu} \lambda_{1}^{n} x+b_{\mu}\left(\sigma_{1}^{n} y-1\right)+\theta_{1}\left(\mu, \lambda_{1}^{n} x, \sigma_{1}^{n} y-1\right)\right)
$$

and

$$
\bar{z}=\sigma_{2}^{m}\left(c_{\mu} \lambda_{1}^{n} x+d_{\mu}\left(\sigma_{1}^{n} y-1\right)+\theta_{2}\left(\mu, \lambda_{1}^{n} x, \sigma_{1}^{n} y-1\right)\right) .
$$

In $(\xi, \eta)$-coordinates, the return map is written as

$$
\begin{aligned}
(\xi, \eta) \mapsto & \left(\sigma_{1}^{n} \sigma_{2}^{m} \alpha(\bar{z}-1)+\sigma_{1}^{n} \sigma_{2}^{m} \tilde{H}_{1}(\mu, \bar{w}, \bar{z}-1),\right. \\
& \left.\sigma_{1}^{2 n} \sigma_{2}^{2 m}\left[-y_{0}+\beta(\bar{z}-1)^{2}+\gamma(\mu) \bar{w}+\mu+\tilde{H}_{2}(\mu, \bar{w}, \bar{z}-1)\right]\right),
\end{aligned}
$$

where now

$$
\begin{aligned}
\bar{w}= & \lambda_{2}^{m} \Delta_{\mu}^{(n)}\left(\sigma_{2}^{-m}\right)+a_{\mu} \lambda_{1}^{n} \lambda_{2}^{m} \sigma_{1}^{-n} \sigma_{2}^{-m} \xi+b_{\mu} \lambda_{2}^{m} \sigma_{1}^{-n} \sigma_{2}^{-2 m} \eta \\
& +\lambda_{2}^{m} \theta_{1}\left(\mu, \lambda_{1}^{n}+\lambda_{1}^{n} \sigma_{1}^{-n} \sigma_{2}^{-m} \xi, \sigma_{1}^{n} y_{0}-1+\sigma_{1}^{-n} \sigma_{2}^{-2 m} \eta\right) \\
& -\lambda_{2}^{m} \theta_{1}\left(\mu, \lambda_{1}^{n}, \sigma_{1}^{n} y_{0}-1\right)
\end{aligned}
$$

and

$$
\begin{aligned}
\bar{z}-1= & c_{\mu} \lambda_{1}^{n} \sigma_{1}^{-n} \xi+d_{\mu} \sigma_{1}^{-n} \sigma_{2}^{-m} \eta \\
& +\sigma_{2}^{m} \theta_{2}\left(\mu, \lambda_{1}^{n}+\lambda_{1}^{n} \sigma_{1}^{-n} \sigma_{2}^{-m} \xi, \sigma_{1}^{n} y_{0}-1+\sigma_{1}^{-n} \sigma_{2}^{-2 m} \eta\right) \\
& -\sigma_{2}^{m} \theta_{2}\left(\mu, \lambda_{1}^{n}, \sigma_{1}^{n} y_{0}-1\right)
\end{aligned}
$$

making use of the definition of $y_{0}$. So we get, using the definition of $\mu_{0}$,

$$
(\xi, \eta) \mapsto\left(H_{1}^{(n, m)}(\nu, \xi, \eta), H_{2}^{(n, m)}(\nu, \xi, \eta)\right) \equiv\left(H^{1}(\nu, \xi, \eta), H^{2}(\nu, \xi, \eta)\right),
$$

where

$$
\begin{aligned}
H_{1}(\nu, \xi, \eta) \equiv & \sigma_{1}^{n} \sigma_{2}^{m} \alpha\left[c_{\mu} \lambda_{1}^{n} \sigma_{1}^{-n} \xi+d_{\mu} \sigma_{1}^{-n} \sigma_{2}^{-m} \eta\right. \\
& \left.+\sigma_{2}^{m} \Theta_{2}^{(n, m)}(\nu, \xi, \eta)+\tilde{H}_{1}(\mu, \bar{w}, \bar{z}-1)\right] \\
H_{2}(\nu, \xi, \eta) \equiv & \sigma_{1}^{2 n} \sigma_{2}^{2 m}\left\{\beta\left[c_{\mu} \lambda_{1}^{n} \sigma_{1}^{-n} \xi+d_{\mu} \sigma_{1}^{-n} \sigma_{2}^{-m} \eta+\sigma_{2}^{m} \Theta_{2}^{(n, m)}(\nu, \xi, \eta)\right]^{2}\right. \\
& +\gamma\left[a_{\mu} \lambda_{1}^{n} \lambda_{2}^{m} \sigma_{1}^{-n} \sigma_{2}^{-m} \xi+b_{\mu} \lambda_{2}^{m} \sigma_{1}^{-n} \sigma_{2}^{-2 m} \eta\right. \\
& \left.+\lambda_{2}^{m} \Theta_{1}^{(n, m)}(\nu, \xi, \eta)\right]+\sigma_{1}^{-2 n} \sigma_{2}^{-2 m} \nu \\
& +\left[\sigma_{1}^{-n} \Gamma_{\mu}^{(m)}\left(\lambda_{1}^{n}\right)-\sigma_{1}^{-n} \Gamma_{\mu}^{(m)}\left(\lambda_{1}^{n}\right)\right. \\
& \left.+\gamma(\mu) \lambda_{2}^{m} \Delta_{\mu}^{(n)}\left(\sigma_{2}^{-m}\right)-\gamma\left(\mu_{0}\right) \lambda_{2}^{m} \Delta_{\mu_{0}}^{(n)}\left(\sigma_{2}^{-m}\right)\right] \\
& \left.+\tilde{H}_{2}(\mu, \bar{w}, \bar{z}-1)\right\}
\end{aligned}
$$


and

$$
\begin{aligned}
\Theta_{i}^{(n, m)}(\nu, \xi, \eta)= & \theta_{i}\left(\mu, \lambda_{1}^{n}+\lambda_{1}^{n} \sigma_{1}^{-n} \sigma_{2}^{-m} \xi, \sigma_{1}^{n} y_{0}-1+\sigma_{1}^{-n} \sigma_{2}^{-2 m} \eta\right) \\
& -\theta_{i}\left(\mu, \lambda_{1}^{n}, \sigma_{1}^{n} y_{0}-1\right)
\end{aligned}
$$

for $i=1,2$.

LEMMA 4.1. - Suppose there is $c<1$ small such that $\sigma_{2}^{2 c} \cdot \lambda_{1}<1$ and $\sigma_{1}$ is so small that $\sigma_{1} \cdot\left(\lambda_{2} \cdot \sigma_{2}\right)^{c / 2}<1$. Choose $m=m(n)$ such that

$$
\frac{c}{2} \cdot n \leq m(n) \leq c \cdot n \text {. }
$$

Then, when restricted to compact parts of $\mathbf{R}^{3}$, the maps

$$
(\nu, \xi, \eta) \mapsto\left(\nu, H_{1}^{(n, m)}(\nu, \xi, \eta), H_{2}^{(n, m)}(\nu, \xi, \eta)\right)
$$

converge in the $C^{3}$ topology, as $n \rightarrow \infty$, to the map

$$
(\nu, \xi, \eta) \mapsto\left(\nu, \alpha d_{0} \eta, \beta d_{0}^{2} \eta^{2}+\nu\right) .
$$

Proof. - Observe first that the hypotheses imply

$$
\begin{array}{r}
\sigma_{1}^{n}\left(\lambda_{2} \sigma_{2}\right)^{m(n)} \longrightarrow 0 \text { as } n \rightarrow \infty \\
\sigma_{2}^{2 m(n)} \cdot \lambda_{1}^{n} \longrightarrow 0 \text { as } n \rightarrow \infty
\end{array}
$$

To obtain the claimed convergence, we will make use of (1) and (2), or their weaker versions. We choose a compact part of $\mathbf{R}^{3}$, so that $|(\nu, \xi, \eta)| \leq$ const., where the convergence will take place and let $K$ be a sufficiently large constant (there will be some slight abuse of notation when dealing with $K$ ).

We have to show the following convergences:

1. $\sigma_{1}^{n} \sigma_{2}^{m}\left[c_{\mu} \lambda_{1}^{n} \sigma_{1}^{-n} \xi+d_{\mu} \sigma_{1}^{-n} \sigma_{2}^{-m} \eta+\sigma_{2}^{m} \Theta_{2}^{(n, m)}(\nu, \xi, \eta)\right] \stackrel{C^{3}}{\longrightarrow} d_{0} \eta$;

2. $\sigma_{1}^{2 n} \sigma_{2}^{2 m}\left[a_{\mu} \lambda_{1}^{n} \lambda_{2}^{m} \sigma_{1}^{-n} \sigma_{2}^{-m} \xi+b_{\mu} \lambda_{2}^{m} \sigma_{1}^{-n} \sigma_{2}^{-2 m} \eta\right.$

$$
\left.+\lambda_{2}^{m} \Theta_{1}^{(n, m)}(\nu, \xi, \eta)\right] \stackrel{C^{3}}{\longrightarrow} 0
$$

3. $\sigma_{1}^{2 n} \sigma_{2}^{2 m}\left[\sigma_{1}^{-n} \Gamma_{\mu_{0}}^{(m)}\left(\lambda_{1}^{n}\right)-\sigma_{1}^{-n} \Gamma_{\mu}^{(m)}\left(\lambda_{1}^{n}\right)+\gamma(\mu) \lambda_{2}^{m} \Delta_{\mu}^{(n)}\left(\sigma_{2}^{-m}\right)\right.$

$$
\left.-\gamma\left(\mu_{0}\right) \lambda_{2}^{m} \Delta_{\mu_{0}}^{(n)}\left(\sigma_{2}^{-m}\right)\right] \stackrel{C^{3}}{\longrightarrow} 0
$$

4. $\sigma_{1}^{n} \sigma_{2}^{m} \tilde{H}_{1}(\mu, \bar{w}, \bar{z}-1) \stackrel{C^{3}}{\longrightarrow} 0$;

5. $\sigma_{1}^{2 n} \sigma_{2}^{2 m} \tilde{H}_{2}(\mu, \bar{w}, \bar{z}-1) \stackrel{C^{3}}{\longrightarrow} 0$.

Vol. $15, n^{\circ}$ 5-1998. 
First notice that $\left|\gamma(\mu)-\gamma\left(\mu_{0}\right)\right|,\left|\Gamma_{\mu}^{(m)}\left(\lambda_{1}^{n}\right)-\Gamma_{\mu_{0}}^{(m)}\left(\lambda_{1}^{n}\right)\right|$ and $\mid \Delta_{\mu}^{(n)}\left(\sigma_{2}^{-m}\right)-$ $\Delta_{\mu_{0}}^{(n)}\left(\sigma_{2}^{-m}\right) \mid$ are bounded by const. $\left|\mu-\mu_{0}\right| \leq K \sigma_{1}^{-2 n} \sigma_{2}^{-2 m}$, so the $C^{0}$ convergence in 3 is guaranteed. The derivatives with respect to $\xi$ and $\eta$ are all zero, and all the derivatives with respect to $\nu$ lead to the appearance of a multiplicative extra factor equal to $\sigma_{1}^{-2 n} \sigma_{2}^{-2 m}$, which implies convergence to zero. Thus item 3 is proved. It is easy to see that $d_{\mu} \eta \stackrel{C^{3}}{\longrightarrow} d_{0} \eta$. We also have

$$
\left|\sigma_{1}^{n} \sigma_{2}^{m} \cdot c_{\mu} \lambda_{1}^{n} \sigma_{1}^{-n} \xi\right| \leq K \lambda_{1}^{n} \sigma_{2}^{m} \rightarrow 0,
$$

by (2), and the derivatives of this term go to zero for the same (or even better) reason. The term $\sigma_{1}^{2 n} \sigma_{2}^{2 m} \cdot a_{\mu} \lambda_{1}^{n} \lambda_{2}^{m} \sigma_{1}^{-n} \sigma_{2}^{-m} \xi$ goes to zero in the $C^{3}$ topology, due to the dissipativeness of $p_{1}$ and $p_{2}$, and

$$
\left|\sigma_{1}^{2 n} \sigma_{2}^{2 m} \cdot b_{\mu} \lambda_{2}^{m} \sigma_{1}^{-n} \sigma_{2}^{-2 m} \eta\right| \leq K \lambda_{2}^{m} \sigma_{1}^{n} \rightarrow 0
$$

by (1), together with all the derivatives. It remains to estimate $\Theta_{i}^{(n, m)}$ and its derivatives up to order 3 to complete 1 and 2 . We have

$$
\left|\Theta_{i}^{(n, m)}\right| \leq \text { const. }\left(\lambda_{1}^{n} \sigma_{1}^{-n} \sigma_{2}^{-m}+\sigma_{1}^{-n} \sigma_{2}^{-2 m}\right)^{2} \leq K \sigma_{1}^{-2 n} \sigma_{2}^{-4 m},
$$

since (2) implies $\lambda_{1}^{n}<\sigma_{2}^{-m}$. To simplify the notation, we define

$$
* \equiv\left(\mu, \lambda_{1}^{n}, \sigma_{1}^{n} y_{0}(\mu)-1\right)
$$

and

$$
* * \equiv\left(\mu, \lambda_{1}^{n}+\lambda_{1}^{n} \sigma_{1}^{-n} \sigma_{2}^{-m} \xi, \sigma_{1}^{n} y_{0}(\mu)-1+\sigma_{1}^{-n} \sigma_{2}^{-2 m} \eta\right) .
$$

Then

$$
\begin{gathered}
\partial_{\xi} \Theta_{i}^{(n, m)}(\nu, \xi, \eta)=\lambda_{1}^{n} \sigma_{1}^{-n} \sigma_{2}^{-m} \partial_{x} \theta_{i}(* *), \\
\partial_{\eta} \Theta_{i}^{(n, m)}(\nu, \xi, \eta)=\sigma_{1}^{-n} \sigma_{2}^{-2 m} \partial_{y} \theta_{i}(* *),
\end{gathered}
$$

and

$$
\begin{aligned}
\partial_{\nu} \Theta_{i}^{(n, m)}(\nu, \xi, \eta) \simeq \sigma_{1}^{-2 n} \sigma_{1}^{-2 m}\{ & {\left[\partial_{\mu} \theta_{i}(* *)-\partial_{\mu} \theta_{i}(*)\right] } \\
& \left.+\left[\partial_{y} \theta_{i}(* *)-\partial_{y} \theta_{i}(*)\right] \cdot\left[\sigma_{1}^{n} y_{0}^{\prime}(\mu)-1\right]\right\},
\end{aligned}
$$

where the aproximate equality stands for the negligible terms coming from the derivatives of the eigenvalues as functions of $\nu$. Thus $\left|\partial_{\xi} \Theta_{i}^{(n, m)}\right| \leq$ $K \lambda_{1}^{n} \sigma_{1}^{-n} \sigma_{2}^{-2 m},\left|\partial_{\eta} \Theta_{i}^{(n, m)}\right| \leq K \sigma_{1}^{-n} \sigma_{2}^{-3 m}$ and $\left|\partial_{\nu} \Theta_{i}^{(n, m)}\right| \leq K \sigma_{1}^{-2 n} \sigma_{2}^{-4 m}$. 
Performing similar (and straightforward) estimates, one obtains else $\left|\partial_{r s} \Theta_{i}^{(n, m)}\right| \leq K \sigma_{1}^{-2 n} \sigma_{2}^{-4 m}$ and $\left|\partial_{r s t} \Theta_{i}^{(n, m)}\right| \leq K \sigma_{1}^{-3 n} \sigma_{2}^{-6 m}$, where $r, s, t \in\{\nu, \xi, \eta\}$. Therefore

$$
\sigma_{1}^{n} \sigma_{2}^{m} \cdot \sigma_{2}^{m} \Theta_{2}^{(n, m)}(\nu, \xi, \eta) \stackrel{C^{3}}{\longrightarrow} 0
$$

and

$$
\sigma_{1}^{2 n} \sigma_{2}^{2 m} \cdot \lambda_{2}^{m} \Theta_{1}^{(n, m)}(\nu, \xi, \eta) \stackrel{C^{3}}{\longrightarrow} 0,
$$

using (1) and dissipativeness of $p_{1}$.

To prove 4. and 5. we first have to do estimates on $\bar{w}$ and $\bar{z}-1$. It is easy to see that $|\bar{w}| \leq K \lambda_{2}^{m},|\bar{z}-1| \leq K \sigma_{1}^{-n} \sigma_{2}^{-m}$, $|\mu| \leq$ const. $\left(\sigma_{1}^{-n}+\lambda_{2}^{m}\right) \leq K \sigma_{1}^{-n},\left|\partial_{\nu} \bar{w}\right| \leq K \lambda_{2}^{m} \sigma_{1}^{-2 n} \sigma_{2}^{-2 m},\left|\partial_{\nu} \bar{z}\right| \leq$ $K \sigma_{1}^{-2 n} \sigma_{2}^{-3 m},\left|\partial_{\xi} \bar{w}\right| \leq K \lambda_{1}^{n} \lambda_{2}^{m} \sigma_{1}^{-n} \sigma_{2}^{-m},\left|\partial_{\xi} \bar{z}\right| \leq K \lambda_{1}^{n} \sigma_{1}^{-n},\left|\partial_{\eta} \bar{w}\right| \leq$ $K \lambda_{2}^{m} \sigma_{1}^{-n} \sigma_{2}^{-2 m},\left|\partial_{\eta} \bar{z}\right| \leq K \sigma_{1}^{-n} \sigma_{2}^{-m}$. Also

$$
\begin{aligned}
&\left|\partial_{r s} \bar{w}\right| \leq \text { const. } \lambda_{2}^{m}\left|\partial_{r s} \Theta_{1}^{(n, m)}\right| \leq K \lambda_{2}^{m} \sigma_{1}^{-2 n} \sigma_{2}^{-4 m}, \\
&\left|\partial_{r s} \bar{z}\right| \leq \text { const. } \sigma_{2}^{m}\left|\partial_{r s} \Theta_{2}^{(n, m)}\right| \leq K \sigma_{1}^{-2 n} \sigma_{2}^{-3 m}, \\
&\left|\partial_{r s t} \bar{w}\right| \leq \text { const. } \lambda_{2}^{m}\left|\partial_{r s t} \Theta_{1}^{(n, m)}\right| \leq K \lambda_{2}^{m} \sigma_{1}^{-3 n} \sigma_{2}^{-6 m}, \\
&\left|\partial_{r s t} \bar{z}\right| \leq \text { const. } \sigma_{2}^{m}\left|\partial_{r s t} \Theta_{2}^{(n, m)}\right| \leq K \sigma_{1}^{-3 n} \sigma_{2}^{-5 m} .
\end{aligned}
$$

Now we can go through 4 . and 5 .

$C^{0}$ convergence: Write the Taylor expansion of $\tilde{H}_{1}$ near $(\mu, 0,0)$, having in mind that $\tilde{H}_{1}(\mu, 0,0) \equiv 0$ :

$$
\begin{aligned}
& \tilde{H}_{1}(\mu, \bar{w}, \bar{z}-1)=\partial_{w} \tilde{H}_{1} \cdot \bar{w}+\partial_{z} \tilde{H}_{1} \cdot(\bar{z}-1)+\frac{1}{2} \partial_{w w} \tilde{H}_{1} \cdot \bar{w}^{2} \\
& \quad+\partial_{w z} \tilde{H}_{1} \cdot \bar{w}(\bar{z}-1)+\frac{1}{2} \partial_{z z} \tilde{H}_{1} \cdot(\bar{z}-1)^{2}+R_{2}(\mu, \bar{w}, \bar{z}-1),
\end{aligned}
$$

where

$$
\lim _{(w, z) \rightarrow 0} \frac{R_{2}(\mu, w, z)}{|(w, z)|^{2}}=0
$$

and all the derivatives are taken at $(\mu, 0,0)$. Observe that

$$
\left|\partial_{w} \tilde{H}_{1}\right|,\left|\partial_{w w} \tilde{H}_{1}\right|,\left|\partial_{w z} \tilde{H}_{1}\right|,\left|\partial_{z z} \tilde{H}_{1}\right| \leq K
$$

and

$$
\left|\partial_{z} \tilde{H}_{1}\right| \leq K \mu,
$$

since $\partial_{z} \tilde{H}_{1}(0,0,0)=0$. Hence

$$
\sigma_{1}^{n} \sigma_{2}^{m}\left|\tilde{H}_{1}(\mu, \bar{w}, \bar{z}-1)\right| \longrightarrow 0
$$

Vol. $15, n^{\circ}$ 5-1998. 
by (1), proving the $C^{0}$ convergence of $\sigma_{1}^{n} \sigma_{2}^{m} \tilde{H}_{1}$.

Now write the Taylor expansion of $\tilde{H}_{2}$ near $(\mu, 0,0)$ :

$$
\begin{aligned}
& \tilde{H}_{2}(\mu, \bar{w}, \bar{z}-1)=\frac{1}{2} \partial_{w w} \tilde{H}_{2} \cdot \bar{w}^{2}+\partial_{w z} \tilde{H}_{2} \cdot \bar{w}(\bar{z}-1)+\frac{1}{2} \partial_{z z} \tilde{H}_{2} \cdot(\bar{z}-1)^{2} \\
& \quad+\frac{1}{6} \partial_{w w w} \tilde{H}_{2} \cdot \bar{w}^{3}+\frac{1}{2} \partial_{w w z} \tilde{H}_{2} \cdot \bar{w}^{2}(\bar{z}-1)+\frac{1}{2} \partial_{w z z} \tilde{H}_{2} \cdot \bar{w}(\bar{z}-1)^{2} \\
& \quad+\frac{1}{6} \partial_{z z z} \tilde{H}_{2} \cdot(\bar{z}-1)^{3}+\frac{1}{24} \partial_{w w w w} \tilde{H}_{2} \cdot \bar{w}^{4}+\frac{1}{6} \partial_{w w w z} \tilde{H}_{2} \cdot \bar{w}^{3}(\bar{z}-1) \\
& \quad+\frac{1}{4} \partial_{w w z z} \tilde{H}_{2} \cdot \bar{w}^{2}(\bar{z}-1)^{2}+\frac{1}{6} \partial_{w z z z} \tilde{H}_{2} \cdot \bar{w}(\bar{z}-1)^{3} \\
& \quad+\frac{1}{24} \partial_{z z z z} \tilde{H}_{2} \cdot(\bar{z}-1)^{4}+R_{4}(\mu, \bar{w}, \bar{z}-1)
\end{aligned}
$$

where the null derivatives $\left(\partial_{w} \tilde{H}_{2}\right.$ and $\left.\partial_{z} \tilde{H}_{2}\right)$ have not been written, all derivatives are taken at $(\mu, 0,0)$ and

$$
\lim _{(w, z) \rightarrow 0} \frac{R_{4}(\mu, w, z)}{|(w, z)|^{4}} \rightarrow 0 .
$$

Observe that the derivatives $\left|\partial_{w w} \tilde{H}_{2}\right|,\left|\partial_{w z} \tilde{H}_{2}\right|,\left|\partial_{w w w} \tilde{H}_{2}\right|,\left|\partial_{w w z} \tilde{H}_{2}\right|$, $\left|\partial_{w z z} \tilde{H}_{2}\right|,\left|\partial_{z z z} \tilde{H}_{2}\right|,\left|\partial_{w w w w} \tilde{H}_{2}\right|,\left|\partial_{w w w z} \tilde{H}_{2}\right|,\left|\partial_{w w z z} \tilde{H}_{2}\right|,\left|\partial_{w z z z} \tilde{H}_{2}\right|$, $\left|\partial_{z z z z} \tilde{H}_{2}\right|$ are all bounded by $K$ and

$$
\left|\partial_{z z} \tilde{H}_{2}\right| \leq K \mu
$$

since $\partial_{w} \tilde{H}_{2}(0,0,0)=\partial_{z z} \tilde{H}_{2}(0,0,0)=0$. Therefore

$$
\sigma_{1}^{2 n} \sigma_{2}^{2 m}\left|\tilde{H}_{2}(\mu, w, z-1)\right| \longrightarrow 0
$$

by (1).

$C^{1}$ convergence: We have

$$
\begin{aligned}
\partial_{\xi} \tilde{H}_{1}(\mu, \bar{w}, \bar{z}-1)= & \partial_{\mu} \tilde{H}_{1}(\mu, \bar{w}, \bar{z}-1) \cdot \partial_{\xi} \mu \\
& +\partial_{w} \tilde{H}_{1}(\mu, \bar{w}, \bar{z}-1) \cdot \partial_{\xi} \bar{w}+\partial_{z} \tilde{H}_{1}(\mu, \bar{w}, \bar{z}-1) \cdot \partial_{\xi} \bar{z}
\end{aligned}
$$

As $\partial_{\xi} \mu=0,\left|\partial_{w} \tilde{H}_{1}\right| \leq K,\left|\partial_{z} \tilde{H}_{1}(\mu, \bar{w}, \bar{z}-1)\right| \leq K\left(\lambda_{2}^{m}+\sigma_{1}^{-n}\right)$, using the estimates above we get

$$
\left|\partial_{\xi} \tilde{H}_{1}(\mu, \bar{w}, \bar{z}-1)\right| \leq K \lambda_{1}^{n} \sigma_{1}^{-2 n}
$$

Similarly,

$$
\left|\partial_{\eta} \tilde{H}_{1}(\mu, \bar{w}, \bar{z}-1)\right| \leq K \sigma_{1}^{-2 n} \sigma_{2}^{-m}
$$


and

$$
\mid \partial_{\nu} \tilde{H}_{1}(\mu, \bar{w}, \bar{z}-1) \leq K \sigma_{1}^{-3 n} \sigma_{2}^{-2 m} .
$$

Also note that $\left|\partial_{\mu} \tilde{H}_{2}\right|,\left|\partial_{w} \tilde{H}_{2}\right|,\left|\partial_{z} \tilde{H}_{2}\right| \leq K \sigma_{1}^{-n}$ at $(\mu, \bar{w}, \bar{z}-1)$, since $\partial_{\mu} \tilde{I}_{2}=\partial_{w} \tilde{H}_{2}=\partial_{z} \tilde{I}_{2}=0$ at $(0,0,0)$. So

$$
\left|\partial_{r} \tilde{H}_{2}(\mu, \bar{w}, \bar{z}-1)\right| \leq K \sigma_{1}^{-n}\left[\left|\partial_{r} \mu\right|+\left|\partial_{r} \bar{w}\right|+\left|\partial_{r} \bar{z}\right|\right],
$$

for $r \in\{\nu, \xi, \eta\}$, which implies

$$
\left|\partial_{\nu} \tilde{H}_{2}(\mu, \bar{w}, \bar{z}-1)\right| \leq K \sigma_{1}^{-3 n} \sigma_{2}^{-2 m}
$$

and

$$
\left|\partial_{\xi} \tilde{H}_{2}(\mu, \bar{w}, \bar{z}-1)\right| \leq K \lambda_{1}^{n} \sigma_{1}^{-2 n}
$$

Hence

$$
\sigma_{1}^{n} \sigma_{2}^{m}\left|\partial_{\xi} \tilde{H}_{1}(\mu, \bar{w}, \bar{z}-1)\right| \leq K \lambda_{1}^{n} \sigma_{1}^{-n} \sigma_{2}^{m} \rightarrow 0
$$

by (1),

$$
\begin{gathered}
\sigma_{1}^{n} \sigma_{2}^{m}\left|\partial_{\eta} \tilde{H}_{1}(\mu, \bar{w}, \bar{z}-1)\right| \leq K \sigma_{1}^{-n} \rightarrow 0, \\
\sigma_{1}^{n} \sigma_{2}^{m}\left|\partial_{\nu} \tilde{H}_{1}(\mu, \bar{w}, \bar{z}-1)\right| \leq K \sigma_{1}^{-2 n} \sigma_{2}^{-m} \rightarrow 0, \\
\sigma_{1}^{2 n} \sigma_{2}^{2 m}\left|\partial_{\xi} \tilde{H}_{2}(\mu, \bar{w}, \bar{z}-1)\right| \leq K \lambda_{1}^{n} \sigma_{2}^{2 m} \rightarrow 0,
\end{gathered}
$$

by (2), and

$$
\sigma_{1}^{2 n} \sigma_{2}^{2 m}\left|\partial_{\nu} \tilde{H}_{2}(\mu, \bar{w}, \bar{z}-1)\right| \leq K \sigma_{1}^{-n} \rightarrow 0 .
$$

It remains to show that $\sigma_{1}^{2 n} \sigma_{2}^{2 m} \partial_{\eta} \tilde{H}_{2}(\mu, \bar{w}, \bar{z}-1)$ goes to zero as $n$ goes to infinity. Write

$$
\partial_{\eta} \tilde{H}_{2}(\mu, \bar{w}, \bar{z}-1)=\partial_{\mu} \tilde{H}_{2} \cdot \partial_{\eta} \mu+\partial_{w} \tilde{H}_{2} \cdot \partial_{\eta} \bar{w}+\partial_{z} \tilde{H}_{2} \cdot \partial_{\eta} \bar{z} .
$$

As $\partial_{\eta} \mu=0$ and $\left|\partial_{w} \tilde{H}_{2} \cdot \partial_{\eta} \bar{w}\right| \leq K \sigma_{1}^{-n} \cdot \lambda_{2}^{m} \sigma_{1}^{-n} \sigma_{2}^{-2 m}$, it is enough to prove that

$$
\left|\sigma_{1}^{2 n} \sigma_{2}^{2 m} \partial_{z} \ddot{H}_{2} \cdot \partial_{\eta} \bar{z}\right| \leq K \sigma_{1}^{n} \sigma_{2}^{m}\left|\partial_{z} \check{H}_{2}(\mu, \bar{w}, \bar{z}-1)\right|
$$

goes to zero. But now we proceed as with the Taylor expansion of $\tilde{H}_{1}$, having in mind that $\partial_{z} \tilde{H}_{2}(\mu, 0,0) \equiv 0$,

$$
\left|\partial_{w z} \tilde{H}_{2}\right|,\left|\partial_{w w z} \tilde{H}_{2}\right|,\left|\partial_{w z z} \tilde{H}_{2}\right|,\left|\partial_{z z z} \tilde{H}_{2}\right| \leq K
$$

and

$$
\left|\partial_{z z} \tilde{H}_{2}\right| \leq K \mu
$$

since $\partial_{z z} \tilde{H}_{2}(0,0,0)=0$ and all the derivatives are taken at $(\mu, 0,0)$.

Vol. $15, \mathrm{n}^{\circ}$ 5-1998. 
$C^{2}$ and $C^{3}$ convergence: Differentiating twice $\tilde{H}_{i}, i=1,2$, and observing that $\left|\partial_{r} \mu\right|,\left|\partial_{r} \bar{w}\right| \leq \sigma_{1}^{-2 n} \sigma_{2}^{-2 m}$ and $\partial_{r s} \mu-0, \forall r, s \in\{\nu, \xi, \eta\}$, we obtain

$$
\begin{aligned}
& \left|\partial_{r s} \tilde{H}_{i}(\mu, \bar{w}, \bar{z}-1)\right| \\
& \quad \leq \\
& \quad K \sigma_{1}^{-2 n} \sigma_{2}^{-2 m}\left[\sigma_{1}^{-2 n} \sigma_{2}^{-2 m}+\lambda_{2}^{m}+\sigma_{2}^{-m}+\left|\partial_{r} \bar{z}\right|+\left|\partial_{s} \bar{z}\right|\right] \\
& \quad+\left|\partial_{z z} \tilde{H}_{i}\right| \cdot\left|\partial_{r} \bar{z}\right| \cdot\left|\partial_{s} \bar{z}\right| .
\end{aligned}
$$

Then

$$
\sigma_{1}^{n} \sigma_{2}^{m} \mid \partial_{r s} \tilde{H}_{1}(\mu, \bar{w}, \bar{z}-1) \leq K \sigma_{1}^{-n} \sigma_{2}^{-m} \longrightarrow 0
$$

and

$\sigma_{1}^{2 n} \sigma_{2}^{2 m}\left|\partial_{r s} \tilde{H}_{2}(\mu, \bar{w}, \bar{z}-1)\right| \leq\left[\sigma_{1}^{-n}+\sigma_{2}^{-m}+\left|\partial_{r} \bar{z}\right|+\left|\partial_{s} \bar{z}\right|\right]+K\left|\partial_{z z} \tilde{H}_{2}\right| \longrightarrow 0$,

since $\partial_{z z} \tilde{H}_{2}(0,0,0)=0$.

Let $r_{1}, r_{2}, r_{3} \in\{\nu, \xi, \eta\}, v_{1}=\mu, v_{2}=w, v_{3}=z, \bar{v}_{1}=\mu=\mu(\nu, \xi, \eta)$, $\bar{v}_{2}=\bar{w}=\bar{w}(\nu, \xi, \eta)$ and $\bar{v}_{3}=\bar{z}=\bar{z}(\nu, \xi, \eta)$. The third order derivatives are given by

$$
\begin{aligned}
& \partial_{r_{1} r_{2} r_{3}} \tilde{H}_{i}\left(\bar{v}_{1}, \bar{v}_{2}, \bar{v}_{3}\right)=\sum_{l=1}^{3} \partial_{v_{l}} \tilde{H}_{i} \cdot \partial_{r_{1} r_{2} r_{3}} \bar{v}_{l} \\
& \quad+\sum_{l=1}^{3} \sum_{j=1}^{3} \partial_{v_{l} v_{j}} \tilde{H}_{i} \cdot\left[\partial_{r_{1} r_{3}} \bar{v}_{l} \cdot \partial_{r_{2}} \bar{v}_{j}+\partial_{r_{1} r_{2}} \bar{v}_{j} \cdot \partial_{r_{3}} \bar{v}_{l}+\partial_{r_{2} r_{3}} \bar{v}_{l} \cdot \partial_{r_{1}} \bar{v}_{j}\right] \\
& \quad+\sum_{l=1}^{3} \sum_{j=1}^{3} \sum_{k=1}^{3} \partial_{v_{k} v_{j} v_{l}} \tilde{H}_{i} \cdot \partial_{r_{1}} \bar{v}_{k} \cdot \partial_{r_{2}} \bar{v}_{j} \cdot \partial_{r_{3}} \bar{v}_{l}
\end{aligned}
$$

where all the derivatives of $\tilde{H}_{i}$ are evaluated at $(\mu, \bar{w}, \bar{z}-1)$. As $\left|\partial_{r_{1} r_{2} r_{3}} \bar{v}_{l}\right| \leq$ $K \sigma_{1}^{-3 n} \sigma_{2}^{-5 m},\left|\partial_{r_{1} r_{2}} \bar{v}_{l}\right| \leq K \sigma_{1}^{-2 n} \sigma_{2}^{-3 m}$ and $\left|\partial_{r_{1}} \bar{v}_{l}\right| \leq K \sigma_{1}^{-n} \sigma_{2}^{-m}$, it follows that

$$
\left|\partial_{r_{1} r_{2} r_{3}} \tilde{H}_{i}(\mu, \bar{w}, \bar{z}-1)\right| \leq K \sigma_{1}^{-3 n} \sigma_{2}^{-3 m},
$$

implying the claimed convergence.

Suppose now that $p_{1}$ and $p_{2}$ are periodic points with periods $k_{1}$ and $k_{2}$. Let $\lambda_{1}^{k_{1}}$ and $\sigma_{1}^{k_{1}}$ be the eigenvalues of $D f^{k_{1}}\left(p_{1}\right), \lambda_{2}^{k_{2}}$ and $\sigma_{2}^{k_{2}}$ the eigenvalues of $D f^{k_{2}}\left(p_{2}\right)$. Assume that $f^{k_{1}}$ is linearizable near $p_{1}$ and $f^{k_{2}}$ is linearizable near $p_{2}$. Suppose also that $\lambda_{1}, \sigma_{1}, \lambda_{2}$ and $\sigma_{2}$ satisfy exactly the same conditions of Proposition 3.7, i.e. there is $c<1$ small such that $\sigma_{2}^{2 c} \cdot \lambda_{1}<1$ and $\sigma_{1}$ is so small that $\sigma_{1} \cdot\left(\lambda_{2} \sigma_{2}\right)^{c / 2}<1$. We want to verify that in this case the renormalization performed for fixed points is still possible. 
If $q$ is the point of quadratic tangency between $W^{s}\left(p_{1}\right)$ and $W^{u}\left(p_{2}\right)$, extend the domain of the linearized coordinates of $f^{k_{2}}$ near $p_{2}$ until it meets $f^{-k_{1} k_{2}}(q)$. The other extensions are made as before. Let $n$ be the number of times the orbit spends in the linear region of $p_{1}$, under $f^{k_{1}}$, and $m$ be the number of times spent in the linear region of $p_{2}$, under $f^{k_{2}}$. Now $f^{k_{1} k_{2}}$ is quadratic near $f^{-k_{1} k_{2}}(q)$ and the renormalization is possible over the return map

$$
f_{\mu}^{k_{1} k_{2}} \circ f_{\mu}^{m k_{2}} \circ f_{\mu}^{n k_{1}}
$$

for a generically unfolding family $\left(f_{\mu}\right)_{\mu}$ with $f_{0}=f$. It remains to choose $m$ as a function of $n$ in order to obtain

$$
\begin{aligned}
\sigma_{1}^{n k_{1}} \cdot\left(\lambda_{2} \sigma_{2}\right)^{m(n) k_{2}} & \longrightarrow 0 \text { as } n \rightarrow \infty, \\
\sigma_{2}^{2 m(n) k_{2}} \cdot \lambda_{1}^{n k_{1}} & \longrightarrow 0 \text { as } n \rightarrow \infty .
\end{aligned}
$$

It is enough to take

$$
\frac{c}{2} n k_{1} \leq m(n) k_{2} \leq c n k_{1} .
$$

This is possible since for $n$ large we have $\frac{c}{2} n k_{1}>k_{2}$.

Suppose now that a saddle $p_{0}$ is not $C^{4}$ linearizable. This means that the eigenvalues at $p_{0}$ are resonant, see [14], [15]. We want to show that by an appropriate arbitrarily $C^{\infty}$ small perturbation it is possible to destroy the resonances and turn the point $p_{0}$ in a $C^{4}$ linearizable one. To be more specific, if $p_{0}$ is a saddle of period $k$, then we are interested in a $C^{4}$ change of coordinates that linearizes $f^{k}$ in a neighborhood of $p_{0}$.

The resonant conditions of Sternberg applied to dissipative saddles with eigenvalues $0<|\lambda|<1<|\sigma|$, are translated into the condition

$$
(\lambda \cdot \sigma)^{n}=\sigma^{-m},
$$

for some pair of integers $(n, m)$ with $n, m \geq 1$. If $f$ is $C^{\infty}$ and we desire just $C^{r}$ linearization, for $r<\infty$, it is enough to avoid a finite number of resonances of this kind, i.e. Eq. 5 with $N=N(f, r) \geq n, m \geq 1$ (the function $N(f, r)$ is continuous on $f$ and $N(f, r) \rightarrow \infty$ as $r \rightarrow \infty)$.

Let $p_{0}$ be a saddle of period $k$ and $\lambda, \sigma$ the eigenvalues of $D f^{k}\left(p_{0}\right)$, satisfying $0<|\lambda|<1<|\sigma|$ and $|\lambda \cdot \sigma|<1$. Let $\phi: W \rightarrow \mathbf{R}^{2}$ be a local chart defined in a neighborhood $W$ of $p_{0}$, with $\phi\left(p_{0}\right)=0$. Assume $W$ sufficiently small so that $f^{j} W \cap W=\emptyset$ for all $0<j<k$. Let $\zeta$ be a $C^{\infty}$ function on $\mathbf{R}$ satisfying

$$
\begin{cases}\zeta(s)=0, & s \geq 2 \\ \zeta(s)=1, & s \leq 1 \\ 0 \leq \zeta(s) \leq 1, & \forall s .\end{cases}
$$


Let $B_{\alpha} \subset \phi(W)$ be a ball of radius $\alpha$ centered at the origin and $\tilde{\zeta}=\zeta\left(4 \frac{\|x\|}{\alpha}\right)$. We define the perturbations $f_{t}=t_{t} \circ f$ of $f$, where

$$
\begin{cases}\iota_{t}(x)=x & \text { if } x \in M \backslash W \\ \iota_{t}(x)=\phi^{-1}([1+t \cdot \tilde{\zeta}(|\phi(x)|)] \cdot \phi(x)) & \text { if } x \in W\end{cases}
$$

We claim that for arbitrarily small $0<t<t_{0}$ the function $f_{t}^{k}$ is linearizable near $p_{0}$. First observe that the eigenvalues of $D f_{t}^{k}\left(p_{0}\right)$ are $(1+t) \lambda$ and $(1+t) \sigma$. Choose $t_{0}$ such that for $t<t_{0}, D f_{t}^{k}\left(p_{0}\right)$ is still hyperbolic and dissipative. Let $D_{n}(t)-((1+t) \lambda \cdot(1+t) \sigma)^{n}$ and $S_{m}(t)-((1+t) \sigma)^{-m}$. If $D f^{k}\left(p_{0}\right)$ is non-resonant, then for every pair $(n, m)$ with $1 \leq n, m \leq N$ we have $D_{n}(0) \neq S_{m}(0)$. If $t_{0}$ is small, then $D_{n}(t) \neq S_{m}(t)$ for every $t<t_{0}$, thus $f_{t}^{k}$ is linearizable near $p_{0}$. If $D f^{k}\left(p_{0}\right)$ is resonant, define the sets of pairs of integers

$$
P_{1}=\left\{(n, m) ; 1 \leq n, m \leq N, D_{n}(0)=S_{m}(0)\right\}
$$

and

$$
P_{2}=\left\{(n, m) ; 1 \leq n, m \leq N, D_{n}(0) \neq S_{m}(0)\right\} .
$$

Choose $t_{0}$ small such that for every $t<t_{0}$ and every $(n, m) \in P_{2}$ we have $D_{n}(t) \neq S_{m}(t)$. For $(n, m) \in P_{1}$ we have $D_{n}^{\prime}(0)=2 n D_{n}(0)=$ $2 n S_{m}(0)=-\frac{2 n}{m} S_{m}^{\prime}(0)$. Then, for $t_{0}$ small, every $0<t<t_{0}$ and $(n, m) \in P_{1}$ satisfy $D_{n}(t) \neq S_{m}(t)$, which implies that $f_{t}^{k}$ is linearizable near $p_{0}$.

Consider now a family $\left(f_{\mu}\right)_{\mu} \in I$ where $I$ is an interval and $f_{0}-f$, and let $p_{\mu}$ be the hyperbolic continuation of the saddle $p_{0}, \lambda_{\mu}$ and $\sigma_{\mu}$ the eigenvalues of $D f_{\mu}^{k}\left(p_{\mu}\right)$ and $\phi_{\mu}: W \mapsto \mathbf{R}^{2}$ a $C^{\infty}$ family of local charts defined in $W$ with $\phi\left(p_{\mu}\right)=0$. Define the perturbated families $\left(f_{\mu, t}\right)_{\mu, t}$ by $f_{\mu, t}=\iota_{\mu, t} \circ f_{\mu}$, where

$$
\begin{cases}\iota_{\mu, t}(x)=x & \text { if } x \in M \backslash W \\ \iota_{\mu, t}(x)=\phi_{\mu}^{-1}\left(\left[1+t \cdot \tilde{\zeta}\left(\left|\phi_{\mu}(x)\right|\right)\right] \cdot \phi_{\mu}(x)\right) & \text { if } x \in W\end{cases}
$$

The eigenvalues of $D f_{\mu, t}^{k}\left(p_{\mu}\right)$ are $(1+t) \lambda_{\mu}$ and $(1+t) \sigma_{\mu}$. Let $J=\left[-t_{0}, t_{0}\right]$, for sufficiently small $t_{0}>0$. The following lemma will be useful in Section 6.

LEMMA 4.2. - The set of $t \in J$ such that $f_{\mu, t}^{k}$ is not linearizable around $p_{\mu}$ for a positive Lebesgue measure set of $\mu$-values in $I$ is countable.

The lemma above is a corollary of the following lemma.

LEMMA 4.3. - Let $I, J \subset \mathbf{R}$ be closed intervals, $r: I \times J \exists$ $(\mu, t) \mapsto r(\mu, t) \in \mathbf{R}$ be a $C^{\infty}$ function satisfying $\frac{\partial r}{\partial t}(\mu, t) \geq c>0$, 
$\forall(\mu, t) \in I \times J$. For $t \in J$, let $Z_{t}=\{\mu \in I ; r(\mu, t)=0\}$. Then the set $\mathcal{T}=\left\{\ell \in \mathcal{J} ; m\left(\mathcal{Z}_{t}\right)>0\right\}$ is countable.

Proof. - The condition $\frac{\partial r}{\partial t}(\mu, t) \geq c>0$ implies that $r(\mu, \cdot)$ is an strictly increasing function, for all $\mu \in I$. Hence $Z_{t} \cap Z_{t^{\prime}}=\emptyset$, if $t \neq t^{\prime}$. Let $\mathcal{T}_{n}=\left\{t ; m\left(\mathcal{Z}_{t}\right)>1 / n\right\}$. Then $\mathcal{T}=\bigcup_{n} \mathcal{T}_{n}$ and each $\mathcal{T}_{n}$ is finite. Thus $\mathcal{T}$ is countable.

To prove Lemma 4.2 , just consider the functions

$$
r_{n, m}(\mu, t)=\left((1+t) \lambda_{\mu} \cdot(1+t) \sigma_{\mu}\right)^{n}-\left((1+t) \sigma_{\mu}\right)^{m}, \quad 1 \leq n, m \leq N .
$$

\section{STRANGE ATTRACTORS NEAR THE QUADRATIC FAMILY}

Now we state and comment the important results in [9], [17] about the existence of strange attractors for Hénon-like families of diffeomorphisms. These results are based in the fundamental work of Benedicks and Carleson [1].

Let $\Psi=\left(\Psi_{a}\right)_{a}$ be the family of endomorphisms of $\mathbf{R}^{2}$ given by

$$
\Psi_{a}(x, y)=\left(1-a x^{2}, 0\right) .
$$

We say that $\Phi=\left(\varphi_{a}\right)_{a}$ is a Hénon-like family if

- $\Phi$ is a $C^{r}$ family of $C^{r}$ diffeomorphisms, $r \geq 3$;

- $\|\Phi-\Psi\|_{C^{3}(R)}$ is sufficiently small,

where $R$ is a sufficiently large rectangle in $\mathbf{R} \times \mathbf{R}^{2}$ (say $R=[-4,4] \times$ $\left.[-10,10]^{2}\right)$. Let $m$ be the Lebesgue measure.

THEOREM 5.1 (Mora-Viana). - Let $0<c<\log 2$ and $\Phi=\left(\varphi_{a}\right)_{a}$ be a Hénon-like family. Then, there is $E=E(c, \Phi) \subset(1,2)$, with $m(E)>0$, such that for every $a \in E$ there is a compact, $\varphi_{a}$-invariant set $\Lambda=\Lambda_{a}$ satisfying

1. the stable set of $\Lambda, W^{s}(\Lambda)$, has non-empty interior;

2. there is $z \in \Lambda$ such that

(a) $\left\{\varphi_{a}^{n}\left(z_{1}\right) ; n \geq 0\right\}$ is dense in $\Lambda$;

(b) $\left\|D \varphi_{a}^{n}\left(z_{1}\right) \cdot(1,0)\right\| \geq e^{\text {cn }}$ for all $n \geq 0$.

The set $\Lambda$ of the theorem is called a Henon-like strange attractor.

Further properties of the set $E=E(c, \Phi)$ can be easily derived from the proof of the theorem above:

Vol. $15, n^{\circ}$ 5-1998. 
1. $E$ is constructed from exclusions of parameters of a host interval $\Delta_{0}$ which does not depend on the family $\Phi$;

2. if $\|\Phi-\Psi\|_{C^{3}(R)}$ is small, the interval $\Delta_{0}$ can be choosen near $a=2$; at the same time, the Lebesgue measure of the excluded parameters relative to $\left|\Delta_{0}\right|$ can be made small, i.e.

$$
m(E) \geq(1-\delta)\left|\Delta_{0}\right|
$$

for choosen $\delta>0$;

3. although the interval $\Delta_{0}$ does not depend on $\Phi$, the excluded parameters do; on the other hand, if we consider only a finite number of exclusions, we can see from the proof that they vary continuously with $\Phi$.

From 3 it is easy to conclude the following lemma, which will be useful in the arguments of Section 6.

THEOREM 5.2. - Let $E=E(\Phi) \subset \Delta_{0}$ be the set obtained in [9], [17], in such a way that for $a \in E, \varphi_{a}$ has a strange attractor. Let $I \subset \Delta_{0}$ be an interval such that $m(E \cap I) \geq c|I|$, for $c>0$. Given $\epsilon>0$, for all $\tilde{\Phi}=\left(\tilde{\varphi}_{a}\right)_{a}$ sufficiently near $\Phi$, there exists $\tilde{E}$ such that $m(\tilde{E} \cap I) \geq(c-\epsilon)|I|$ and for $a \in \tilde{E} \tilde{\varphi}_{a}$ has a strange attractor.

When we look at the unfolding $\left(f_{\mu}\right)_{\mu}$ of the 2-cycle involving $p_{1}$ and $p_{2}$ considered in Section 4 , we see a sequence of host intervals $\Delta_{m, n}$ in the $\mu$-space, going to zero as $n$ and $m$ tend to infinity, each one corresponding to $\Delta_{0}$ by the $(n, m)$-change of coordinates. Moreover, if we embed the family $\left(f_{\mu}\right)_{\mu}$ in a $C^{\infty}$ two-parameter family $\left(f_{\mu, \beta}\right)_{\mu, \beta}$, we find, for each $\beta$ sufficiently small, a sequence $\Delta_{m, n}(\beta)$ of host intervals converging to $\mu_{T}(\beta)$, the value of tangency between $W^{s}\left(p_{1}\right)$ and $W^{u}\left(p_{2}\right)$. It is easy to see that $\Delta_{m, n}(\beta)$ depends continuously on $\beta$, since the $(n, m)$-parameter changes of the families $\left(f_{\mu, \beta}\right)_{\mu}$ do. Also, the convergence of the families in Lemma 4.1 is uniform in $\beta$, since all bounding constants can be taken the same for all families $\left(\int_{\mu, \beta}\right)_{\mu}$ with $\beta$ small. So for each $\beta$ small, there is a set $E_{n, m}(\beta) \subset \Delta_{n, m}(\beta)$ of parameters presenting strange attractors, as a direct application of the theorem of Mora-Viana.

Altogether, these assumptions imply that we can fix $\beta_{0}$ small and obtain: "given $\epsilon>0$, there are $n_{0}=n_{0}\left(\beta_{0}\right), m_{0}=m_{0}\left(\beta_{0}\right)$ such that for all $\Delta_{n, m}(\beta)$ with $n \geq n_{0}, m \geq m_{0}$ and $\beta<\beta_{0}$, we have:

- $\sup \left\{\left|\mu-\mu_{T}(\beta)\right| ; \mu \in \Delta_{n, m}(\beta)\right\}<\epsilon$;

- $m\left(E_{n, m}(\beta) \cap \Delta_{n, m}(\beta)\right) \geq \frac{3}{4}\left|\Delta_{n, m}(\beta)\right|$;

- $\Delta_{n, m}(\beta)$ is continuous with respect to $\beta$." 


\section{PROOF OF THE MAIN RESULTS}

\subsection{Preliminary remarks}

Let $f_{0} \in \operatorname{Diff}^{\infty}(M)$ be such that $p$ is a dissipative periodic saddle point with a homoclinic tangency between its stable and unstable manifolds. Hence there is $f \in \operatorname{Diff}^{\infty}(M) C^{\infty}$ arbitrarily near $f_{0}$ with hyperbolic sets $\Lambda_{1}, \Lambda_{2}$ and points $Q_{1} \in \Lambda_{1}, Q_{2} \in \Lambda_{2}$ satisfying items 1 to 3 of Proposition 3.7. Let $\mathcal{U} \subset \operatorname{Diff}^{\infty}(M)$ be a sufficiently small neighborhood of $f$. For $g \in \mathcal{U}$, there are hyperbolic continuations $\Lambda_{1}(g)$ and $\Lambda_{2}(g)$ of $\Lambda_{1}$ and $\Lambda_{2}$, respectively. That is, there exist $C^{\infty}$ functions

$$
\begin{aligned}
\Phi_{i}: \mathcal{U} & \longrightarrow C^{0}\left(\Lambda_{i}, M\right), i=1,2 \\
g & \longmapsto \Phi_{i}(g)
\end{aligned}
$$

such that $\Lambda_{i}(g)=\Phi_{i}(g)\left(\Lambda_{i}\right)$ is a basic set for $g$, where $C^{0}\left(\Lambda_{i}, M\right)$ is the space of injective continuous functions from $\Lambda_{i}$ into $M$. Moreover, $\Phi_{i}(g)$ conjugates $\left.f\right|_{\Lambda_{i}}$ to $\left.g\right|_{\Lambda_{i}(y)}$.

For $x \in \Lambda_{i}$, denote by $W^{s}(x, g)$ the stable manifold $W^{s}\left(\Phi_{i}(g) x, g\right)$ and $W^{u}(x, g)$ the unstable manifold $W^{u}\left(\Phi_{i}(g) x, g\right)$ of the continuation $\Phi_{i}(g) x$ of $x$. We know that, as a $C^{\infty}$ embedded disk, $W_{\mathrm{loc}}^{s}(x, g)$ varies continuously with $x$ and is a $C^{\infty}$ function of $g$. Hence, there are small balls $B_{\delta}\left(Q_{1}\right)$ and $B_{\delta}\left(Q_{2}\right)$, centered at $Q_{1}$ and $Q_{2}$, such that for all $g \in \mathcal{U}, x \in B_{\delta}\left(Q_{1}\right) \cap \Lambda_{1}, y \in B_{\delta}\left(Q_{2}\right) \cap \Lambda_{2}, W^{u}(x, g)$ and $W^{s}(y, g)$ meet transversally in a neighborhood of $r$, the point of transversal intersection between $W^{u}\left(Q_{1}\right)$ and $W^{s}\left(Q_{2}\right)$ mentioned in Proposition 3.7.

Let $U$ be a sufficiently small neighborhood of $q$, the point of quadratic tangency between $W^{s}\left(Q_{1}\right)$ and $W^{u}\left(Q_{2}\right)$ ( $U$ will be eventually diminished in order to satisfy further requirements). Put $C^{\infty}$ coordinates $(u, v) \in[-1,1]^{2}$ in $U$ in such a way that

1. $q$ has coordinates $(0,0)$;

2. the connected component of $W^{s}\left(Q_{1}\right) \cap U$ containing $q$ is given by $\{v=0\}$;

3. for $x \in B_{\delta}\left(Q_{1}\right) \cap \Lambda_{1}$ and $g \in \mathcal{U}$, the connected component of $W^{s}(x, g) \cap U$ which corresponds in the obvious way to $W^{s}\left(Q_{1}\right) \cap U$ is given by $\left\{v=A_{1}(x)(u, g)\right\}$;

4. for $y \in B_{\delta}\left(Q_{2}\right) \cap \Lambda_{2}$ and $g \in \mathcal{U}$, the connected component of $W^{u}(y, g) \cap U$ corresponding in the obvious way to the connected component of $W^{u}\left(Q_{2}\right) \cap U$ containing $q$ is given by $\{v=$ $\left.A_{2}(y)(u, g)\right\}$

5. $A_{2}\left(Q_{2}\right)(0, f)=0=\partial_{u} A_{2}\left(Q_{2}\right)(0, f)$. 
In view of the above discussion,

$$
x \mapsto A_{i}(x)([-1,1], g)
$$

is continuous in the $C^{\infty}$ topology and

$$
A_{i}(x):[-1,1] \times \mathcal{U} \rightarrow[-1,1]
$$

is $C^{\infty}, i=1,2$. From the hypothesis of quadratic tangency between $W^{s}\left(Q_{1}\right)$ and $W^{u}\left(Q_{2}\right)$, we may assume

$$
\partial_{u u}\left(A_{2}(y)-A_{1}(x)\right)(u, g) \geq c>0
$$

for any $u \in[-1,1], g \in \mathcal{U}, x \in B_{\delta}\left(Q_{1}\right) \cap \Lambda_{1}$ and $y \in B_{\delta}\left(Q_{2}\right) \cap \Lambda_{2}$, where $c$ is a sufficiently small constant. As a consequence, all possible tangencies between $A_{1}$-leaves and $A_{2}$-leaves in $U$ for $g \in \mathcal{U}$ are quadratic.

\subsection{Control of orbits}

Suppose there are periodic points $p_{1} \in B_{\delta}\left(Q_{1}\right) \cap \Lambda_{1}$ and $p_{2} \in B_{\delta}\left(Q_{2}\right) \cap \Lambda_{2}$ such that for $g \in \mathcal{U}, W^{s}\left(p_{1}, g\right)$ and $W^{u}\left(p_{2}, g\right)$ are tangent inside $U$. Since $W^{u}\left(p_{1}, g\right)$ and $W^{s}\left(p_{2}, g\right)$ meet transversally near $r$, we can apply the renormalization scheme of Section 4 to find, for $\tilde{g}$ near $g$, a strange attractor of very high period. It will be important in 6.4 that we have some control on the orbit of this strange attractor. To be more specific, we will require that the orbit of the strange attractor intersects $U$ only once, implying that any perturbation done inside $U$ but outside a neighborhood of the strange attractor does not affect the remaining of the orbit.

Let $U^{\prime}$ be a neighborhood of $r$. Define $L_{1}^{u}\left(Q_{1}\right)$ to be the least closed segment of $W^{u}\left(Q_{1}\right)$ from $Q_{1}$ through $r$ that crosses $U^{\prime}$ and for $x \in B_{\delta}\left(Q_{1}\right) \cap \Lambda_{1}$ let $L_{1}^{u}(x)$ be the least closed segment of $W^{u}(x, f)$ that corresponds in the obvious way to $L_{1}^{u}\left(Q_{1}\right)$ and crosses $U^{\prime}$. Analogously define $L_{2}^{s}\left(Q_{2}\right)$ and $L_{2}^{s}(y)$, for $y \in B_{\delta}\left(Q_{2}\right) \cap \Lambda_{2}$, and replacing $U^{\prime}$ by $U$, make the natural definitions of $L_{1}^{s}\left(Q_{1}\right), L_{1}^{s}(x), L_{2}^{u}\left(Q_{2}\right)$ and $L_{2}^{u}(y)$. Define also $L_{1}^{s} \equiv \bigcup_{x \in B_{\delta}\left(Q_{1}\right) \cap \Lambda_{1}} L_{1}^{s}(x), L_{1}^{u} \equiv \bigcup_{x \in B_{\delta}\left(Q_{1}\right) \cap \Lambda_{1}} L_{1}^{u}(x)$, $L_{2}^{s} \equiv \bigcup_{y \in B_{\delta}\left(Q_{2}\right) \cap \Lambda_{2}} L_{2}^{s}(y)$, and $L_{2}^{u} \equiv \bigcup_{y \in B_{b}\left(Q_{2}\right) \cap \Lambda_{2}} L_{2}^{u}(y)$. Choose $\delta>0$ in such a way that $B_{\delta}\left(Q_{1}\right) \cap \Lambda_{1} \cap W_{\operatorname{loc}\left(Q_{1}\right)}^{s}, B_{\delta}\left(Q_{1}\right) \cap \Lambda_{1} \cap W_{\operatorname{loc}\left(Q_{1}\right)}^{u}$, $B_{\delta}\left(Q_{2}\right) \cap \Lambda_{2} \cap W_{\text {loc }\left(Q_{2}\right)}^{s}$ and $B_{\delta}\left(Q_{2}\right) \cap \Lambda_{2} \cap W_{\text {loc }\left(Q_{2}\right)}^{u}$ are compact. Hence $L_{1}^{s}, L_{1}^{u}, L_{2}^{s}$ and $L_{2}^{u}$ are compact. Let $U_{1}$ and $U_{2}$ be neighborhoods of $\Lambda_{1}$ and $\Lambda_{2}$ and take $l_{0}$ such that $f^{l}\left(L_{1}^{s}\right) \cup f^{-l}\left(L_{1}^{u}\right) \subset U_{1}$ and $f^{l}\left(L_{2}^{s}\right) \cup f^{-l}\left(L_{2}^{u}\right) \subset U_{2}$ 
for all $l \geq l_{0}$. Consider the compact set

$$
\begin{aligned}
\Omega= & \overline{\left(W_{\mathrm{loc}}^{s}\left(\Lambda_{1}\right) \cup W_{\mathrm{loc}}^{u}\left(\Lambda_{1}\right)\right) \cap U_{1}} \cup \bigcup_{j=1}^{l_{0}} f^{j}\left(L_{1}^{s}\right) \cup \bigcup_{j=0}^{l_{0}} f^{-j}\left(L_{1}^{u}\right) \\
& \cup \overline{\left(W_{\mathrm{loc}}^{s}\left(\Lambda_{2}\right) \cup W_{\mathrm{loc}}^{u}\left(\Lambda_{2}\right)\right) \cap U_{2}} \cup \bigcup_{j=0}^{l_{0}} f^{j}\left(L_{2}^{s}\right) \cup \bigcup_{j=1}^{l_{0}} f^{-j}\left(L_{2}^{u}\right)
\end{aligned}
$$

and its natural continuation $\Omega_{g}$ for $g \in \mathcal{U}$, which is clearly defined since $\Omega$ is made up by arcs of stable and unstable manifolds.

Suppose there are periodic points $p_{1} \in B_{\delta}\left(Q_{1}\right) \cap \Lambda_{1}$ and $p_{2} \in B_{\delta}\left(Q_{2}\right) \cap \Lambda_{2}$ such that for $g \in \mathcal{U}, W^{s}\left(p_{1}, g\right)$ and $W^{u}\left(p_{2}, g\right)$ have a (quadratic) tangency inside $U$. Assume $f^{k_{1}}$ linearizable around $p_{1}, f^{k_{2}}$ linearizable around $p_{2}$, where $k_{1}$ and $k_{2}$ are the periods of $p_{1}$ and $p_{2}$. Take a family $\left(g_{\mu}\right)_{\mu} \subset \mathcal{U}$ with $g_{0}=g$ generically unfolding the quadratic tangency. By Sections 4 and 5 , there are a sequence of host intervals $\Delta_{n} \rightarrow 0$, subsets $E_{n} \subset \Delta_{n}$ with $m\left(E_{n}\right)>0$ and integers $k_{n} \rightarrow \infty$ as $n \rightarrow \infty$ such that for $\mu \in E_{n}$, $g_{\mu}^{k_{n}}$ has a strange attractor $S_{n}=S_{n}(\mu)$ inside $U$. Moreover, given $\alpha>0$, there is $n_{0}$ sufficiently large such that

$$
\left\{g_{\mu}\left(S_{n}\right), g_{\mu}^{2}\left(S_{n}\right), \ldots, g_{\mu}^{k_{n}-1}\left(S_{n}\right)\right\} \subset B_{\alpha}\left(\Omega_{g}\right)
$$

for all $n \geq n_{0}$, where $B_{\alpha}\left(\Omega_{g}\right)$ denotes the $\alpha$-neighborhood of $\Omega_{g}$.

We claim that if $U, \mathcal{U}, \delta$ and $\alpha$ are sufficiently small, then in fact

$$
g_{\mu}^{j}\left(S_{n}\right) \cap U=\emptyset, \quad \forall 0<j<k_{n} .
$$

For that, it is enough to choose $U, \mathcal{U}$ and $\alpha$ in such a way that $B_{\alpha}\left(\Omega_{g}\right) \cap U=\emptyset$ ( $\delta$ is chosen after $U$ in order to satisfy item 4 of 6.1 ). If $f(U) \cap U=\emptyset$ and $f^{-1}(U) \cap U=\emptyset$ then $q \notin \Omega$. $\Lambda \mathrm{s} \Omega$ is compact and composed by stable and unstable manifolds, if $U$ is sufficiently small then $U \cap \Omega=\emptyset$. This implies that there are $\alpha$ and $\mathcal{U}$ such that $B_{\alpha}\left(\Omega_{g}\right) \cap U=\emptyset$ for all $g \in \mathcal{U}$.

\subsection{The line of tangency}

For each $g \in \mathcal{U}$, there is a $C^{1}$ foliation defined in the whole of $U$ which extends the leaves of $\Lambda_{i}$. In other words, for $i=1,2$, there is a $C^{1}$ vector field $X_{i}=X_{i}(g):[-1,1]^{2} \rightarrow \mathbf{R}^{2}$ such that

1. $g \mapsto X_{i}(g)(u, v)$ is $C^{\infty}$, for each $(u, v) \in[-1,1]^{2}$;

2. $X_{i}(g)\left(u_{0}, A_{i}(x)\left(u_{0}, g\right)\right)$ is colinear with $\left(u_{0}, \partial_{u} A_{i}(x)\left(u_{0}, g\right)\right), \forall u_{0} \in$ $[-1,1], g \in \mathcal{U}, x \in \Lambda_{i}$. 
Since $X_{1}(f)(0,0) \cdot X_{2}(f)(0,0)^{\perp}=0$ and the tangency is quadratic, by the Implicit Function Theorem there is a $C^{1}$ line $l_{g}$ such that for $(u, v) \in l_{g}$ we have

$$
X_{1}(g)(u, v) \cdot X_{2}(g)(u, v)^{\perp}=0 .
$$

The line $l_{g}$ is called the line of tangency for $g$ (see Figure 4).

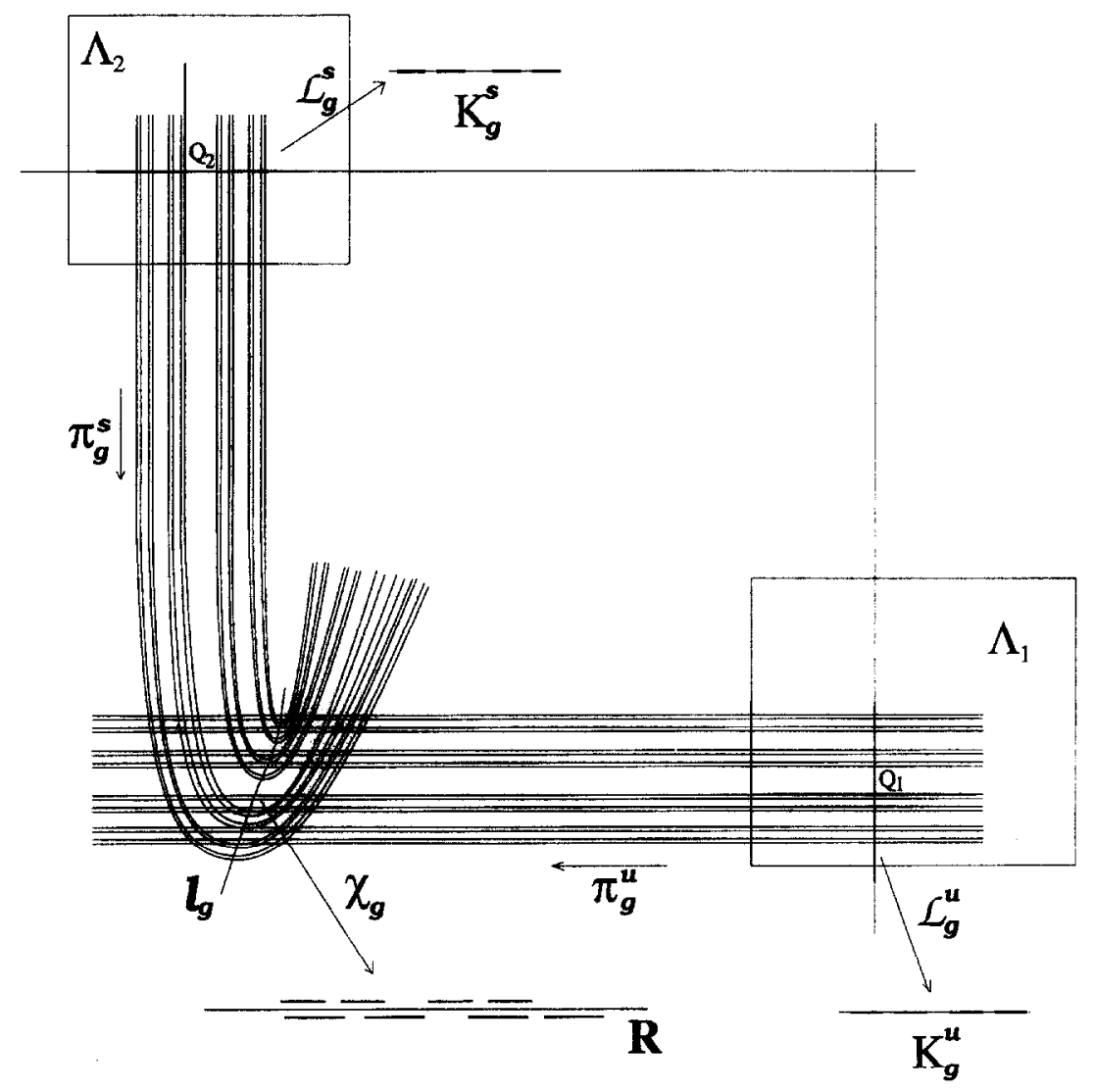

Fig. 4. - The line of tangency.

Let $\pi_{g}^{u}: W_{\mathrm{loc}}^{u}\left(Q_{1}, g\right) \rightarrow l_{g}$ (resp. $\left.\pi_{g}^{s}: W_{\mathrm{loc}}^{s}\left(Q_{2}, g\right) \rightarrow l_{g}\right)$ be the $C^{1}$ projection along stable (resp. unstable) leaves of $\Lambda_{1}$ (resp. $\Lambda_{2}$ ). Let $\chi_{g}: l_{g} \rightarrow \mathbf{R}$ be a $C^{1}$ parametrization of the line of tangency. Consider also $C^{\infty}$ parametrizations $\mathcal{L}_{g}^{u}: W^{u}\left(Q_{1}, g\right) \rightarrow \mathbf{R}$ and $\mathcal{L}_{g}^{s}: W^{s}\left(Q_{2}, g\right) \rightarrow \mathbf{R}$ with $\mathcal{L}_{g}^{u}\left(\Phi_{1}(g) Q_{1}\right)=\mathcal{L}_{g}^{s}\left(\Phi_{2}(g) Q_{2}\right)=0$. Define the Cantor sets

$$
K_{g}^{u}=\mathcal{L}_{g}^{u}\left(W_{\mathrm{loc}}^{u}\left(Q_{1}, g\right) \cap \Lambda_{1}(g)\right)
$$


and

$$
K_{g}^{s}=\mathcal{L}_{g}^{s}\left(W_{\mathrm{loc}}^{s}\left(Q_{2}, g\right) \cap \Lambda_{2}(g)\right),
$$

which are dynamically defined (see [12]) and are near $K_{f}^{u}$ and $K_{f}^{y}$, respectively, if $g$ is near $f$. The function $\mathcal{L}_{g}^{u} \circ \Phi_{1}(g) \circ\left(\mathcal{L}_{f}^{u}\right)^{-1}: K_{f}^{u} \rightarrow K_{g}^{u}$ (resp. $\mathcal{L}_{g}^{s} \circ \Phi_{2}(g) \circ\left(\mathcal{L}_{f}^{s}\right)^{-1}: K_{f}^{s} \rightarrow K_{g}^{s}$ ) is the natural equivalence between $K_{f}^{u}$ (resp. $K_{f}^{s}$ ) and its continuation $K_{g}^{u}$ (resp. $K_{g}^{s}$ ).

It follows from Proposition 3.7 that there is some $t>0$ such that

$$
\tau_{\text {loc }}\left(K_{f}^{u}\right) \cdot \tau_{\text {loc }}\left(K_{f}^{s}\right) \geq 1+t
$$

The definition of local thickness and the equicontinuity of thickness (see Section 2) imply that for $\mathcal{U}$ small enough, there exists $\epsilon_{0}>0$ such that for any $\epsilon_{0}>\epsilon>0$ we can find Cantor sets $\tilde{K}_{f}^{u} \subset K_{f}^{u} \cap B_{\epsilon}(0)$ and $\tilde{K}_{f}^{s} \subset K_{f}^{s} \cap B_{\epsilon}(0)$ whose continuations $\tilde{K}_{g}^{u}$ and $\tilde{K}_{g}^{s}$ satisfy

$$
\tau_{\text {loc }}\left(\tilde{K}_{g}^{u}\right) \cdot \tau_{\text {loc }}\left(\tilde{K}_{g}^{s}\right) \geq 1+\frac{3 t}{4}, \forall g \in \mathcal{U}
$$

If $\epsilon_{0}$ and $\mathcal{U}$ are small enough, we still obtain

$$
\tau\left(\chi_{g} \circ \pi_{g}^{u} \circ\left(\mathcal{L}_{g}^{u}\right)^{-1}\left(\tilde{K}_{g}^{u}\right)\right) \cdot \tau\left(\chi_{g} \circ \pi_{g}^{s} \circ\left(\mathcal{L}_{g}^{s}\right)^{-1}\left(\tilde{K}_{g}^{s}\right)\right) \geq 1+\frac{t}{2},
$$

by Proposition 2.3 .

Now we are seeking to determine open sets $\mathcal{W} \subset \operatorname{Diff}^{\infty}(M)$ arbitrarily close to $f_{0}$ such that for all $g \in \mathcal{W}$ there is a tangency between $W^{s}\left(\Lambda_{1}\right)$ and $W^{u}\left(\Lambda_{2}\right)$. A set like $\mathcal{W}$ is often called an open set of persistent tangencies. First define the functions

$$
\begin{aligned}
\vartheta_{g}^{u}: K_{f}^{u} & \longrightarrow \mathbf{R} \\
x & \longmapsto \chi_{g} \circ \pi_{g}^{u} \circ \Phi_{1}(g) \circ\left(\mathcal{L}_{f}^{u}\right)^{-1}(x)
\end{aligned}
$$

and

$$
\begin{aligned}
\vartheta_{g}^{s}: K_{f}^{s} & \longrightarrow \mathbf{R} \\
x & \longmapsto \chi_{g} \circ \pi_{g}^{s} \circ \Phi_{2}(g) \circ\left(\mathcal{L}_{f}^{s}\right)^{-1}(x),
\end{aligned}
$$

which can be extended to the convex hull of $K_{f}^{u}$ and $K_{f}^{s}$. Let $\left(\hat{f}_{\mu}\right)_{\mu \in[-1,+1]} \subset \mathcal{U}$ be a $C^{\infty}$ family of diffeomorphisms generically unfolding the tangency between $W^{u}\left(Q_{1}, f\right)$ and $W^{s}\left(Q_{2}, f\right)$. To be more specific, we require that $\hat{f}_{0}=f$ and

$$
\partial_{\mu}\left[A_{1}(x)\left(u, \hat{f}_{\mu}\right)-A_{2}(y)\left(u^{\prime}, \hat{f}_{\mu}\right)\right] \geq c>0,
$$

Vol. $15, n^{\circ}$ 5-1998. 
for any $x \in \Lambda_{1}, y \in \Lambda_{2}, u, u^{\prime} \in[-1,1]$ and $\mu \in[-1,1]$. As a consequence of (6), for any $x \in K_{f}^{u}, y \in K_{f}^{s}$, we have

$$
\partial_{\mu}\left[\vartheta_{\hat{f}_{\mu}}^{u}(x)-\vartheta_{\hat{f}_{\mu}}^{s}(y)\right] \geq c>0,
$$

if $c$ is small enough.

Consider the Cantor sets $\tilde{K}_{f}^{u} \subset K_{f}^{u} \cap B_{\epsilon}(0)$ and $\tilde{K}_{f}^{s} \subset K_{f}^{s} \cap B_{\epsilon}(0)$ introduced above, with the property that $\tau\left(\vartheta_{g}^{u}\left(\tilde{K}_{f}^{u}\right)\right) \cdot \tau\left(\vartheta_{g}^{s}\left(\tilde{K}_{f}^{s}\right)\right) \geq 1+t / 2$, for any $\epsilon_{0}>\epsilon>0, g \in \mathcal{U}, \epsilon_{0}$ and $\mathcal{U}$ small. Then, by $(\mathcal{T})$, there is a $\mu_{0}$ such that

- $\left|\mu_{0}\right|<2 c^{-1} \epsilon$;

- the pair $\left\langle\vartheta_{\hat{f}_{\mu}}^{u}\left(\tilde{K}_{f}^{u}\right), \vartheta_{\hat{f}_{\mu}}^{s}\left(\tilde{K}_{f}^{s}\right)\right\rangle$ is linked for $\mu=\mu_{0}$.

Let $\mathcal{Z}$ be a neighborhood of $F=\left(\hat{f}_{\mu}\right)_{\mu}$ in the space of one-parameter families and $I$ an interval around $\mu_{0}$. Taking $\mathcal{Z}$ and $I$ sufficiently small, and defining for $G=\left(g_{\mu}\right)_{\mu} \in \mathcal{Z}$ the Cantor sets $K^{u}(G ; \mu) \equiv \vartheta_{g_{\mu}}^{u}\left(\tilde{K}_{f}^{u}\right)$ and $K^{s}(G ; \mu) \equiv \vartheta_{g_{\mu}}^{s}\left(\tilde{K}_{f}^{s}\right)$, then we have that $\left\langle K^{s}(G ; \mu), K^{u}(G ; \mu\rangle\right.$ is a linked pair for $\mu \in I$, since the linking property is an open condition.

Define

$$
\mathcal{W}=\left\{g_{\mu} ; G=\left(g_{\mu}\right)_{\mu} \in \mathcal{Z}, \mu \in I\right\} .
$$

This set corresponds to one of the sets $\mathcal{W}_{n}$ mentioned in the Introduction. We still obtain, by equicontinuity of thickness, that if $C_{u}$ is a bridge of $\tilde{K}_{f}^{u}$ and $C_{s}$ is a bridge of $\tilde{K}_{f}^{s}$, then for any $g \in \mathcal{W}$,

$$
\tau\left(\vartheta_{g}^{u}\left(C_{u} \cap \tilde{K}_{f}^{u}\right)\right) \cdot \tau\left(\vartheta_{g}^{s}\left(C_{s} \cap \tilde{K}_{f}^{s}\right)\right) \geq 1+\frac{t}{2}
$$

Moreover, (6) can be stated for any $G=\left(g_{\mu}\right)_{\mu} \in \mathcal{Z}$ :

$$
\partial_{\mu}\left[A_{1}(x)\left(u, g_{\mu}\right)-A_{2}(y)\left(u^{\prime}, g_{\mu}\right)\right] \geq c>0
$$

for any $x \in \Lambda_{1}, y \in \Lambda_{2}, u, u^{\prime} \in[-1,+1]$ and $\mu \in[-1,+1]$.

\subsection{The argument}

Claim: Given any subinterval $I^{\prime} \subset I$, there exists a residual subset $\mathcal{R}$ of $\mathcal{Z}$ such that for each family $G=\left(g_{\mu}\right)_{\mu} \in \mathcal{R}$, there is a parameter $\mu_{\infty}(G) \in I^{\prime}$ for which $g_{\mu_{\infty}(G)}$ exhibits infinitely many strange attractors.

The Claim clearly implies the existence of a residual subset $\tilde{\mathcal{R}} \subset \mathcal{Z}$ such that for each family $G=\left(g_{\mu}\right)_{\mu} \in \tilde{\mathcal{R}}$, the set of parameters $\mu \in I$ for which $g_{\mu}$ has infinitely many strange attractors is dense in $I$. So, Theorem A 
is proved, and Theorem B follows easily from the remark at the end of Section 3.

The proof of the Claim will be done by induction. Let $U$ be as in 6.2 and $\mathcal{Z} \supset \mathcal{R}_{1} \supset \ldots \supset \mathcal{R}_{N} \supset \ldots$ be a sequence of sets satisfying

1. for all $N \geq 1$ and each family $G=\left(g_{\mu}\right)_{\mu \in I} \in \mathcal{R}_{N}$, there is a compact set $E_{N}=E_{N}(G) \subset I^{\prime}, m\left(E_{N}\right)>0$, such that for $\mu \in E_{N}, g_{\mu}$ has $N$ distinct strange attractors $S_{1}=S_{1}(G ; \mu), \ldots, S_{N}=S_{N}(G ; \mu)$; furthermore,

(a) for all $i=1, \ldots, N$, the strange attractor $S_{i}$ is generated by renormalization (see 6.2) and the orbit of $S_{i}$ intersects $U$ only once, inside $B_{r_{i}}$, where $B_{r_{i}} \subset U$ is a ball of radius $r_{i}$, and $B_{r_{i}} \cap B_{r_{j}}=\emptyset$ for all $i \neq j$;

(b) $E_{N+1}(G) \subset E_{N}(G)$, for $N \geq 1$;

2. for each $G \in \mathcal{R}_{N}$ and $\mu$ in a neighborhood of the convex hull of $E_{N}(G)$, there are bridges $P_{i}^{s}$ of $\tilde{K}_{f}^{s}$ and $P_{i}^{u}$ of $\tilde{K}_{f}^{u}, i=1, \ldots, N$, such that

(a) their images $P_{i}^{s}(G ; \mu) \equiv \vartheta_{g_{\mu}}^{s}\left(P_{i}^{s}\right)$ and $P_{i}^{u}(G ; \mu) \equiv \vartheta_{g_{\mu}}^{u}\left(P_{i}^{u}\right)$ form a stable linked pair (see Figure 5);

(b) their images on the line of tangency $\tilde{P}_{i}^{s}(G ; \mu) \equiv \chi_{g_{\mu}}^{-1}\left(P_{i}^{s}(G ; \mu)\right)$ and $\tilde{P}_{i}^{u}(G ; \mu) \equiv \chi_{g_{\mu}}^{-1}\left(P_{i}^{u}(G ; \mu)\right)$ satisfy

$$
\tilde{P}_{i}^{s}(G ; \mu) \cap \tilde{P}_{i}^{u}(G ; \mu) \subset B_{r_{i}} ;
$$

3. for each $G \in \mathcal{R}_{N}$ and $\mu$ in a neighborhood of the convex hull of $E_{N}(G)$, there are bridges $Q_{N}^{s}$ of $\tilde{K}_{f}^{s}$ and $Q_{N}^{u}$ of $\tilde{K}_{f}^{u}$ such that

(a) their images $Q_{N}^{s}(G ; \mu) \equiv \vartheta_{g_{\mu}}^{s}\left(Q_{N}^{s}\right)$ and $Q_{N}^{u}(G ; \mu) \equiv \vartheta_{g_{\mu}}^{u}\left(Q_{N}^{u}\right)$ form a linked pair;

(b) their images on the line of tangency $\tilde{Q}_{N}^{s}(G ; \mu) \equiv \chi_{g_{\mu}}^{-1}\left(Q_{N}^{s}(G ; \mu)\right)$ and $\tilde{Q}_{N}^{u}(G ; \mu) \equiv \chi_{g_{\mu}}^{-1}\left(Q_{N}^{u}(G ; \mu)\right)$ satisfy

$$
\tilde{Q}_{N}^{s}(G ; \mu) \cap \tilde{Q}_{N}^{u}(G ; \mu) \subset B_{\epsilon},
$$

where $B_{\epsilon} \subset U$ is a ball of radius $\epsilon$ and $B_{\epsilon} \cap B_{r_{i}}=\emptyset$, for any $i=1, \ldots, N$.

We will show that $\mathcal{R}_{N \mid 1}$ is open and dense in $\mathcal{R}_{N}$. This will imply that the set $\mathcal{R}=\bigcap_{N>0} \mathcal{R}_{N}$ is residual and for each $G=\left(g_{\mu}\right)_{\mu} \in \mathcal{R}$ there is a nested sequence $I^{\prime} \supset E_{1}(G) \supset E_{2}(G) \supset \ldots \supset E_{N}(G) \supset \ldots$ of compact sets as in the item 1 of the induction. Hence if $\mu_{\infty}(G) \in \bigcap_{N \geq 0} E_{N}(G) \subset I^{\prime}$, then $g_{\mu_{\infty}(G)}$ has infinitely many strange attractors. 

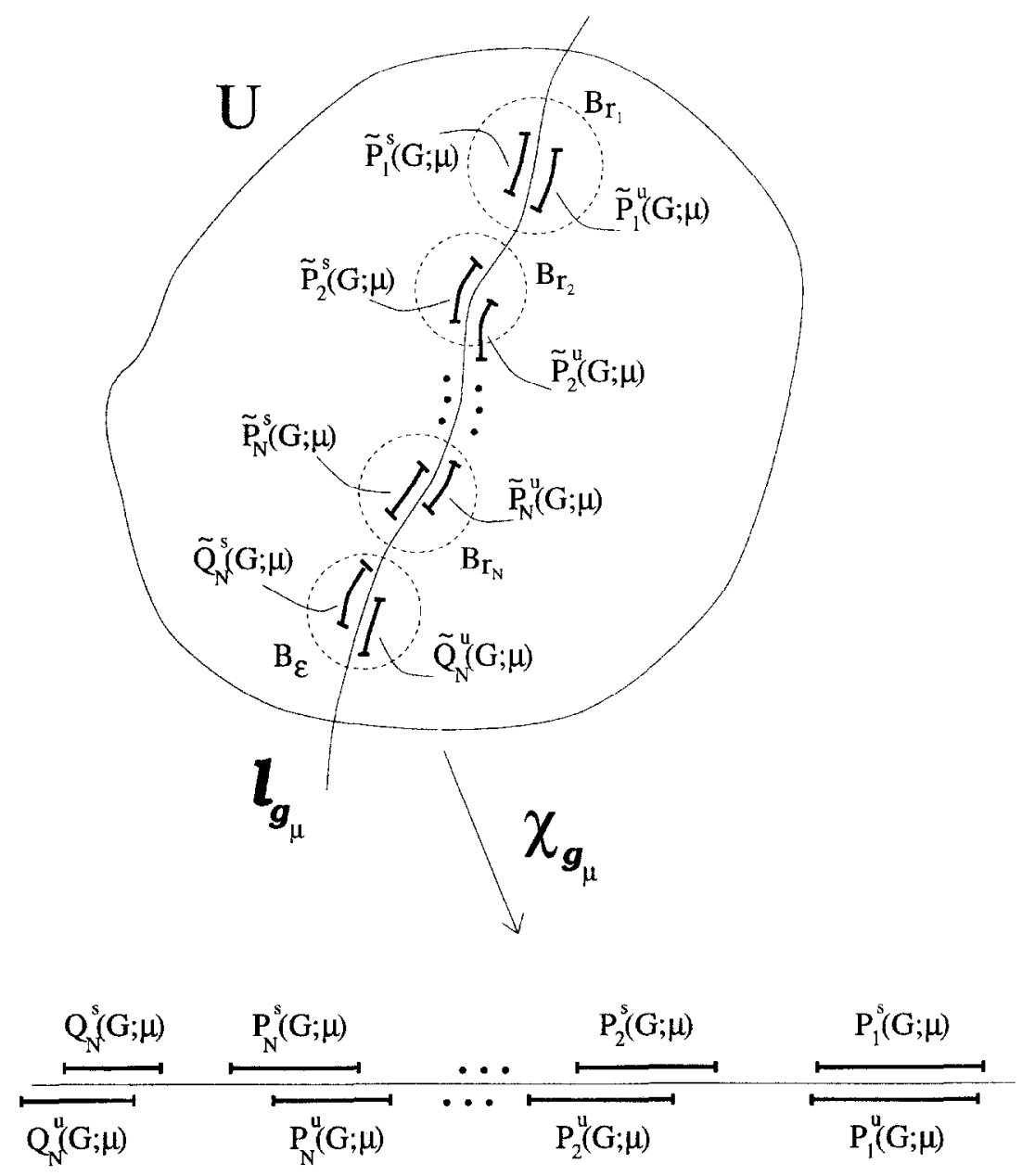

Fig. 5. - Induction hypotheses.

Now we prove that $\mathcal{R}_{N+1}$ is dense in $\mathcal{R}_{N}, N \geq 1$. The openess of $\mathcal{R}_{N}$, $N \geq 1$ is an easy consequence of Lemma 5.2 .

Let $G=\left(g_{\mu}\right)_{\mu} \in \mathcal{R}_{N}, N \geq 1$. We will show by successive perturbations that there exists $H=\left(h_{\mu}\right)_{\mu} \in \mathcal{R}_{N+1} C^{\infty}$ arbitrarily near $G$ (the proof also shows that $\mathcal{R}_{1}$ is dense in $\mathcal{Z}$; for that, take $G \in \mathcal{Z}, E_{0}(G)=I^{\prime}$, $Q_{0}^{s}$ the convex hull of $\tilde{K}_{f}^{s}, Q_{0}^{u}$ the convex hull of $\tilde{K}_{f}^{u}$ and proceed as below with $N=0$ ).

First perturbation. Choose a density point of $E_{N}$, i.e. a point $\mu_{N}$ such that

$$
\lim _{\rho \rightarrow 0} \frac{m\left(E_{N} \cap\left[\mu_{N}-\rho, \mu_{N}+\rho\right]\right)}{2 \rho}=1 .
$$


Consider the bump function $\zeta$ of Section 4 . Let $\gamma_{1}$ be less than half the distance from $\bigcup_{\mu \in E_{N}} \tilde{Q}_{N}^{s}(G ; \mu) \cap \tilde{Q}_{N}^{u}(G ; \mu)$ to $\mathbf{R}^{2} \backslash B_{\epsilon}$. We will define $G^{(1)}=\left(g_{\mu}^{(1)}\right)_{\mu}$ such that $g_{\mu}^{(1)}=g_{\mu}$ out of $B_{\epsilon}$, for each $\mu$, and there are two (stable) sublinks associated to the linked pair $\left\langle Q_{N}^{u}\left(G^{(1)} ; \mu_{N}\right), Q_{N}^{s}\left(G^{(1)} ; \mu_{N}\right)\right\rangle$. For that, let $q_{1}$ be the center of $B_{\epsilon}$ and let

$$
\zeta_{1}(u, v)=\zeta\left(\frac{3}{\gamma_{1}} \cdot\left(\left\|(u, v)-q_{1}\right\|-\left(\epsilon-\gamma_{1}\right)\right)\right) .
$$

Define, for $\beta$ small, the $C^{\infty}$ diffeomorphism

$$
\begin{aligned}
T_{\beta}: M & \longrightarrow M \\
(u, v) & \longmapsto\left(u, v+\beta \cdot \zeta_{1}(u, v)\right) \text { in } U, \\
x & \longmapsto x \quad \text { in } M \backslash U .
\end{aligned}
$$

Denote by $T_{\beta} \circ G$ the family $\left(T_{\beta} \circ g_{\mu}\right)_{\mu}$. Since $\left\langle Q_{N}^{s}\left(G ; \mu_{N}\right), Q_{N}^{u}\left(G ; \mu_{N}\right)\right\rangle$ is a linked pair by the induction hypotheses and the Cantor sets $Q_{N}^{s}\left(T_{\beta} \circ G ; \mu_{N}\right) \cap K^{s}\left(T_{\beta} \circ G ; \mu_{N}\right), Q_{N}^{u}\left(T_{\beta} \circ G ; \mu_{N}\right) \cap K^{u}\left(T_{\beta} \circ G ; \mu_{N}\right)$ have non-zero $\beta$-velocity with respect to each other, all the hypotheses of the Linking Lcmma are satisfied. Therefore we obtain $\beta_{1}$ arbitrarily small for which there are two disjoint sublinks associated to the link above. We have

$$
\left\|T_{\beta_{1}} \circ G-G\right\|_{C^{r}} \leq \text { const. } \mid \beta_{1}\left\|\zeta_{1}\right\|_{C^{r}} \leq \text { const. }\left|\beta_{1}\right|\left(\frac{3}{\gamma_{1}}\right)^{r}
$$

and, by 6.2 , the perturbation inside $B_{\epsilon}$ does not affect the links of item 2 of the induction.

Define $G^{(1)} \equiv T_{\beta_{1}} \circ G$ and let

$$
\begin{aligned}
& \left\langle P_{N+1}^{s}\left(G^{(1)} ; \mu_{N}\right), P_{N+1}^{u}\left(G^{(1)} ; \mu_{N}\right)\right\rangle, \\
& \left\langle Q_{N+1}^{s}\left(G^{(1)} ; \mu_{N}\right), Q_{N+1}^{u}\left(G^{(1)} ; \mu_{N}\right)\right\rangle
\end{aligned}
$$

be the sublinks of $\left\langle Q_{N}^{s}\left(G^{(1)} ; \mu_{N}\right), Q_{N}^{u}\left(G^{(1)} ; \mu_{N}\right)\right\rangle$, where

$$
\begin{aligned}
& P_{N+1}^{s, u}\left(G^{(1)} ; \mu\right) \equiv \vartheta_{g_{\mu}^{(1)}}^{s, u}\left(P_{N+1}^{s, u}\right), \\
& Q_{N+1}^{s, u}\left(G^{(1)} ; \mu\right) \equiv \vartheta_{g_{\mu}^{(1)}}^{s, u}\left(Q_{N+1}^{s, u}\right)
\end{aligned}
$$

for some bridges $P_{N+1}^{s}, Q_{N+1}^{s}$ of $\tilde{K}_{f}^{s}, P_{N+1}^{u}, Q_{N+1}^{u}$ of $\tilde{K}_{f}^{u}$. Let $B_{r_{N+1}} \subset B_{\epsilon}$ be a ball of radius $r_{N+1}$ containing $\tilde{P}_{N+1}^{s}\left(G^{(1)} ; \mu_{N}\right) \cap \tilde{P}_{N+1}^{u}\left(G^{(1)} ; \mu_{N}\right)$ and $B_{\epsilon^{\prime}} \subset B_{\epsilon}$ be a ball containing $\tilde{Q}_{N+1}^{s}\left(G^{(1)} ; \mu_{N}\right) \cap \tilde{Q}_{N+1}^{u}\left(G^{(1)} ; \mu_{N}\right)$, with $B_{\epsilon^{\prime}} \cap B_{r_{N+1}}=\emptyset$. 
Second perturbation: Since the pair

$$
\left\langle P_{N+1}^{s}\left(G^{(1)} ; \mu_{N}\right), P_{N+1}^{u}\left(G^{(1)} ; \mu_{N}\right)\right\rangle
$$

is linked, there is a tangency between a stable leaf of $\Lambda_{1}$, say $A_{1}(x)\left(\cdot, g_{\mu_{N}}^{(1)}\right)$, $x \in \Lambda_{1}$, and an unstable leaf of $\Lambda_{2}$, say $A_{2}(y)\left(\cdot, g_{\mu_{N}}^{(1)}\right), y \in \Lambda_{2}$. Given $d_{1}, d_{2}>0$, there are periodic points $p_{1} \in B_{s}\left(Q_{1}\right) \cap \Lambda_{1}$ and $p_{2} \in B_{s}\left(Q_{2}\right) \cap \Lambda_{2}$ such that

$$
\begin{aligned}
& \left|A_{1}\left(p_{1}\right)\left(u, g_{\mu_{N}}^{(1)}\right)-A_{1}(x)\left(u, g_{\mu_{N}}^{(1)}\right)\right| \leq d_{1}, \\
& \left|A_{2}\left(p_{2}\right)\left(u, g_{\mu_{N}}^{(1)}\right)-A_{2}(y)\left(u, g_{\mu_{N}}^{(1)}\right)\right| \leq d_{2},
\end{aligned}
$$

for every $u \in[-1,1]$. Let $k_{1}$ and $k_{2}$ be the periods of $p_{1}$ and $p_{2}$. Fixing $\eta>0$ small and making a perturbation similar to the one described in Section 4 , we obtain a family $G^{(2)}=\left(g_{\mu}^{(2)}\right)_{\mu}$ near $G^{(1)}$ such that $\left(g_{\mu}^{(2)}\right)^{k_{1}}$ is $C^{4}$ linearizable near $p_{1}$ and $\left(g_{\mu}^{(2)}\right)^{k_{2}}$ is $C^{4}$ linearizable near $p_{2}$ for Lebesgue almost every point $\mu \in\left[\mu_{N}-\eta, \mu_{N}+\eta\right]$. As $G^{(2)}$ can be chosen arbitrarily near $G^{(1)}$ independently from $\eta$, we use Lemma 5.2 to conclude that there is a density point of $E_{N}\left(G^{(2)}\right)$, say $\mu_{N}^{\prime}$, with $\left|\mu_{N}^{\prime}-\mu_{N}\right| \leq \eta$ and the additional property that $\left(g_{\mu_{N}^{\prime}}^{(2)}\right)^{k_{1}}$ is $C^{4}$ linearizable near $p_{1}$ and $\left(g_{\mu_{N}^{\prime}}^{(2)}\right)^{k_{2}}$ is $C^{4}$ linearizable near $p_{2}$.

Observe that the choice of $p_{1}, p_{2}$ and the perturbation from $G^{(1)}$ to $G^{(2)}$ can be done in such a way that $W^{s}\left(p_{1}, g_{\mu_{N}^{\prime}}^{(2)}\right)$ and $W^{u}\left(p_{2}, g_{\mu_{N}^{\prime}}^{(2)}\right)$ cross the line of tangency inside $B_{T_{N+1}-2 \gamma_{2}}$, where $B_{r_{N+1}-2 \gamma_{2}}$ is the ball of radius $r_{N+1}-2 \gamma_{2}$ concentric with $B_{r_{N+1}}$ and $\gamma_{2}>0$ is a small constant. Moreover, if the perturbation is sufficiently small, the link $\left\langle Q_{N+1}^{s}\left(G^{(1)} ; \mu_{N}\right), Q_{N+1}^{u}\left(G^{(1)} ; \mu_{N}\right)\right\rangle$ is not destroyed, i.e. $\left\langle Q_{N+1}^{s}\left(G^{(2)} ; \mu_{N}^{\prime}\right), Q_{N+1}^{u}\left(G^{(2)} ; \mu_{N}^{\prime}\right)\right\rangle$ is a linked pair and $Q_{N+1}^{s}\left(G^{(2)} ; \mu_{N}^{\prime}\right) \cap$ $Q_{N+1}^{u}\left(G^{(2)} ; \mu_{N}^{\prime}\right) \subset B_{\epsilon^{\prime}}$. Finally, we may assume that

$$
\begin{aligned}
& \left|A_{1}\left(p_{1}\right)\left(u, g_{\mu_{N}^{\prime}}^{(2)}\right)-A_{1}\left(p_{1}\right)\left(u, g_{\mu_{N}}^{(1)}\right)\right| \leq \eta, \\
& \left|A_{2}\left(p_{2}\right)\left(u, g_{\mu_{N}^{\prime}}^{(2)}\right)-A_{2}\left(p_{2}\right)\left(u, g_{\mu_{N}}^{(1)}\right)\right| \leq \eta,
\end{aligned}
$$

and that $d_{1}+d_{2}+3 \eta$ is small with respect to $\gamma_{2}$.

Third perturbation. Let $q_{2}$ be the center of $B_{r_{N+1}}$ and

$$
\zeta_{2}(u, v)=\zeta\left(\frac{3}{\gamma_{2}} \cdot\left(\left\|(u, v)-q_{2}\right\|-\left(r_{N+1}-\gamma_{2}\right)\right)\right) .
$$

As in the first perturbation define, for $\beta$ small, the $C^{\infty}$ diffeomorphism

$$
\begin{aligned}
\tilde{T}_{\beta}: M & \longrightarrow M \\
(u, v) & \longmapsto\left(u, v+\beta \cdot \zeta_{2}(u, v)\right) \text { in } U, \\
x & \longmapsto x \text { in } M \backslash U .
\end{aligned}
$$


Hence there exists a $\beta_{2}$ with $\left|\beta_{2}\right| \leq$ const. $\left(d_{1}+d_{2}+3 \eta\right)$ such that $W^{s}\left(p_{1}, \tilde{T}_{\beta_{2}} \circ g_{\mu_{N}^{\prime}}^{(2)}\right)$ and $W^{u}\left(p_{2}, \tilde{T}_{\beta_{2}} \circ g_{\mu_{N}^{\prime}}^{(2)}\right)$ have a tangency inside $B_{r_{N+1}-\gamma_{2}}$.

Define $G^{(3)}=\left(g_{\mu}^{(3)}\right)_{\mu} \equiv \tilde{T}_{\beta_{2}} \circ G^{(2)}$, using the same notation as before, and observe that $p_{1}$ and $p_{2}$ are still $C^{4}$ linearizable for $\left(g_{\mu_{N}^{\prime}}^{(3)}\right)^{k_{1}}$ and $\left(g_{\mu_{N}^{\prime}}^{(3)}\right)^{k_{2}}$, respectively, since $G^{(3)}=G^{(2)}$ out of $B_{r_{N+1}}$. Furthermore, $E_{N}\left(G^{(3)}\right)=E_{N}\left(G^{(2)}\right)$, since the orbits of the strange attractors $S_{i}$, $i=1, \ldots, N$, intersect $U$ only out of $B_{\epsilon} \supset B_{r_{N+1}}$. As a consequence, $\mu_{N}^{\prime}$ is still a density point of $E_{N}\left(G^{(3)}\right)$. Also,

$$
\left\|G^{(3)}-G^{(2)}\right\|_{C^{r}} \leq \text { const. }\left|\beta_{2}\right|\left(\frac{3}{\gamma_{2}}\right)^{r} .
$$

Fourth perturbation. Now we consider $\tilde{T}_{\beta} \circ G^{(3)}=\tilde{T}_{\rho_{2}+\rho} \circ G^{(2)}$. Let $\mu_{T}(\beta)$ be such that $W^{s}\left(p_{1}, \tilde{T}_{\beta} \circ g_{\mu_{T}(\beta)}^{(3)}\right)$ and $W^{u}\left(p_{2}, \tilde{T}_{\beta} \circ g_{\mu_{T}(\beta)}^{(3)}\right)$ are tangent. Clearly $\mu_{T}(0)=\mu_{N}^{\prime}$. Let $\Delta$ be a host interval of strange attractors in the $\mu$-space given by the renormalization scheme involving $p_{1}$ and $p_{2}$ for the family $G^{(3)}$, and let $\Delta(\beta)$ be its natural continuation for the family $\tilde{T}_{\beta} \circ G^{(3)}$, as already discussed in Section 5. These intervals exist since all families are inside $\mathcal{W}$ and (8) is valid, implying generic unfolding. By Section 5 , the relative measure of the set $E(\beta)$ of strange attractors in $\Delta(\beta)$ can be taken greater than $\frac{3}{4}$, and $\Delta$ can be chosen in such a way that $\Delta(\beta)$ is arbitrarily near $\mu_{T}(\beta)$, uniformly with $\beta$. We may suppose, without loss of generality, that $\Delta(\beta)$ is on the right of $\mu_{T}(\beta)$, for $\beta$ small, and $\mu_{T}(\beta)$ decreases as $\beta$ increases. As a consequence, we can choose $\beta_{3}>0$ arbitrarily small and $\Delta$ so near $\mu_{N}^{\prime}$ that

$$
\mu_{T}\left(\beta_{3}\right)<\mu<\mu_{T}(0)=\mu_{N}^{\prime}, \forall \mu \in \Delta\left(\beta_{3}\right)
$$

Hence, if we denote by $\mu_{\Delta}(\beta)$ the center of the interval $\Delta(\beta)$, there is $0<\beta_{4}<\beta_{3}$ such that $\mu_{\Delta}\left(\beta_{4}\right)=\mu_{T}(0)=\mu_{N}^{\prime}$. Since $E_{N}\left(\tilde{T}_{\beta} \circ G^{(3)}\right)=$ $E_{N}\left(G^{(3)}\right), \forall \beta$, using 6.2 , and $\mu_{N}^{\prime}$ is a density point of $E_{N}\left(G^{(3)}\right)$, there is $\rho_{0}$ such that for all $\rho \leq \rho_{0}$,

$$
m\left(E_{N}\left(\tilde{T}_{\beta} \circ G^{(3)}\right) \cap\left[\mu_{N}^{\prime}-\rho, \mu_{N}^{\prime}+\rho\right]\right) \geq \rho .
$$

Imposing $|\Delta(\beta)|<\rho_{0}$ in the choice of $\Delta$, it follows that

$$
m\left(E_{N}\left(\check{T}_{\beta_{4}} \circ G^{(3)}\right) \cap E\left(\beta_{4}\right)\right) \geq\left(\frac{3}{4}-\frac{1}{2}\right)\left|\Delta\left(\beta_{4}\right)\right|>0 .
$$


Defining $H=\tilde{T}_{\beta_{4}} \circ G^{(3)}$, it is clear that $H$ belongs to $\mathcal{R}_{N+1}$, with $E_{N+1}(H)=E_{N}(I I) \cap E\left(\beta_{4}\right) \subset E_{N}(H)$, again by 6.2. Furthermore,

$$
\left\|H-G^{(3)}\right\|_{C^{r}} \leq \text { const. }\left|\beta_{4}\right|\left(\frac{3}{\gamma_{2}}\right)^{r} \text {, }
$$

which implies

$$
\begin{aligned}
\|H-G\|_{C^{r}} \leq & \text { const. }\left(\left|\beta_{1}\right|\left(\frac{3}{\gamma_{1}}\right)^{r}+\left|\beta_{2}\right|\left(\frac{3}{\gamma_{2}}\right)^{r}\right. \\
& \left.+\left|\beta_{4}\right|\left(\frac{3}{\gamma_{2}}\right)^{r}\right)+\left\|G^{(2)}-G^{(1)}\right\|_{C^{r}} .
\end{aligned}
$$

As $\beta_{1}$ is taken arbitrarily small with respect to $\gamma_{1}, \beta_{2}$ and $\beta_{4}$ are also small with respect to $\gamma_{2}$ and $\left\|G^{(2)}-G^{(1)}\right\|_{C^{r}}$ is arbitrarily small for any $r$, by Section 4 , we conclude that $\|H-G\|_{C^{r}}$ can be arbitrarily small for any $r$.

The Claim is proved.

For the proof of Theorem $\mathrm{C}$, instead of considering, in the induction, a positive measure set $F_{N}$ at the paramater space for which the family $G$ exhibits $N$ strange attractors, just consider a parameter value $\mu_{N}$ for which there are $N$ phenomena of $\Sigma$ type. The perturbation from $G$ to $G^{(1)}$ is identical, but the perturbation from $G^{(1)}$ to $G^{(2)}$ causes to change the parameters for which the $N$ phenomena of $\Sigma$ type appear. If they become separated in the parameter space, hy making a perturbation similar to the third and fourth perturbations above in each $B_{r_{i}}, i=1, \ldots, N$, with size proportional to the size of $G^{(2)}-G^{(1)}$, one obtains a family $\tilde{G}^{(2)}$ such that for $\tilde{g}_{\mu_{N}}^{(2)}$ there are $N$ phenomena of $\Sigma$ type. As these perturbations are done inside $U$, they do not affect the eigenvalues of $p_{1}$ and $p_{2}$. After that, one applies identically the third perturbation. To apply the fourth perturbation, it is enough to substitute the host intervals $\Delta(\beta)$ by single parameter values, where the new phenomena of $\Sigma$ type are generated. The openess of $\mathcal{R}_{N}$ is not possible when considering codimension-one phenomena. Thus, the Claim is translated into the following statement: "Given any subinterval $I^{\prime} \subset I$, there exists a dense set $\mathcal{R}$ of $\mathcal{Z}$ such that for each family $G=\left(g_{\mu}\right)_{\mu} \in \mathcal{R}$, there is a parameter $\mu_{\infty}(G) \in I^{\prime}$ for which $g_{\mu_{\infty}(G)}$ exhibits infinitely many phenomena of $\Sigma$ type." This assertion obviously imply Theorem $C$.

\section{AKNOWLEDGEMENTS}

I am specially grateful to Prof. J. Palis for posing me the problem and for his encouragement in all stages of this work. I am also grateful to my 
colleagues at IMPA for many pleasant discussions and valuable suggestions. Finally, I aknowledge financial support from CNPq during the preparation of this work.

\section{REFERENCES}

[1] M. Benedicks and L. Carleson, The dynamics of the Hénon map, Ann. Math., Vol. 133, 1991, pp. 73-169.

[2] E. CATSIGERAS, Cascades of period doubling of stable codimension one, Thesis IMPA, 1995.

[3] J.-M. Gambaudo and C. Tresser, Diffeomorphisms with infinitely many strange attractors, J. of Complexity, Vol. 6, 1990, pp. 409-416.

[4] R. Kraft, Intersection of thick Cantor sets, Mem. Amer. Math. Soc., Vol. 97, No. 468(2), 1992, pp. 1-119.

[5] S. Newhouse, Non-density of Axiom A(a) on $S^{2}$, Proc. A.M.S. Symp. Pure Math., Vol. 14, 1970, pp. 191-202.

[6] S. NEwHouSE, Diffeomorphisms with infinitely many sinks, Topology, Vol. 13, 1974, pp. 9-18.

[7] S. NewhousE, The abundance of wild hyperbolic sets and nonsmooth stable sets for diffeomorphisms, Publ. Math. I.H.E.S., Vol. 50, 1979, pp. 101-151.

[8] S. Newhouse, J. Palis and F. TAKENS, Bifurcations and stability of families of diffeomorphisms, Publ. Math. I.H.E.S., Vol. 57, 1983, pp. 5-71.

[9] L. MORA and M. VIANA, Abundance of strange attractors, Acta Math., Vol. 171, 1993, pp. 1-71.

[10] H. Poincare, Sur le problème des trois corps et les équations de la dynamique (Mémoire couronné du prise de S.M. le roi Oscar II de Suède), Acta Math., Vol. 13, 1890, pp. 1-270.

[11] A. Pumariño and J. A. Rodriguez, Coexistence and persistence of strange attractors, 1994, to appear.

[12] J. PALIS and F. TAKens. Hyperbolicity and sensitive chaotic dynamics at homoclinic bifurcations, Cambridge University Press, 1993.

[13] C. Robinson, Bifurcation to infinitely many sinks, Comm. Math. Phys., Vol. 90, 1983, pp. $433-459$.

[14] S. Sternberg, Local contractions and a theorem of Poincaré, Amer. J. Math., Vol. 79, 1957, pp. 809-824.

[15] S. Sternberg, On the structure of local homeomorphisms of Euclidean $n$-space, II, Amer. J. Math., Vol. 80, 1958, pp. 623-631.

[16] R. URES, Approximating a Hénon-like strange attractor by a homoclinic tangency and an attracting cycle, to appear in Erg. Th. and Dyn. Syst.

[17] M. VIANA, Strange attractors in higher dimensions, Bol. Soc. Bras. Mat., Vol. 24(1), 1993 , pp. 13-62.

[18] J. A. YORKE and K. T. AlligoOD, Cascades of period doubling bifurcations: a pre-requisite for horseshoes, Bull. A.M.S., Vol. 9, 1983, pp. 319-322.

(Manuscript received April 29, 1996.)

Vol. $15, n^{\circ} 5-1998$ 